\title{
Generalized Meir-Keeler type $n$-tupled fixed point theorems in ordered partial metric spaces
}

\author{
Mohammad Imdad ${ }^{1 *}$, Anupam Sharma ${ }^{1}$ and Ali Erduran ${ }^{2}$
}

\section{"Correspondence:}

mhimdad@yahoo.co.in

'Department of Mathematics,

Aligarh Muslim University, Aligarh,

202002, India

Full list of author information is

available at the end of the article

\begin{abstract}
In this paper, we prove $n$-tupled fixed point theorems (for even $n$ ) for mappings satisfying Meir-Keeler type contractive condition besides enjoying mixed monotone property in ordered partial metric spaces. As applications, some results of integral type are also derived. Our results generalize the corresponding results of Erduran and Imdad (J. Nonlinear Anal. Appl. 2012:jnaa-00169, 2012).
\end{abstract}

Keywords: partially ordered set; partial metric space; generalized Meir-Keeler type contractive condition; mixed monotone property; $n$-tupled fixed point

\section{Introduction}

Existence of a fixed point for contraction type mappings in partially ordered metric spaces with possible applications have been considered recently by many authors (e.g. [1-31]). Recently many researchers have obtained fixed and common fixed point results on partially ordered metric spaces (see $[5,10,11,18,22,32,33])$. In 2006, Bhaskar and Lakshmikantham [13] initiated the idea of coupled fixed point and proved some interesting coupled fixed point theorems for mappings satisfying a mixed monotone property. In this continuation, Lakshmikantham and Ćirić [17] generalized these results for nonlinear $\phi$-contraction mappings by introducing two ideas namely: coupled coincidence point and mixed $g$-monotone property. Thereafter Samet and Vetro [34] extended the idea of coupled fixed point to higher dimensions by introducing the notion of fixed point of $n$-order (or $n$-tupled fixed point, where $n$ is natural number greater than or equal to 2 ) and presented some $n$-tupled fixed point results in complete metric spaces, using a new concept of $F$-invariant set. On the other hand, Imdad et al. [35] generalized the idea of $n$-tupled fixed point by considering even-tupled coincidence point besides exploiting the idea of mixed $g$-monotone property on $X^{n}$ and proved an even-tupled coincidence point theorem for nonlinear $\phi$-contraction mappings satisfying mixed $g$-monotone property.

The concept of partial metric space was introduced by Matthews [36] in 1994, which is a generalization of usual metric space. In such spaces, the distance of a point to itself may not be zero. The main motivation behind the idea of a partial metric space is to transfer mathematical techniques into computer science. Following this initial work, Matthews [36] generalized the Banach contraction principle in the context of complete partial metric spaces. For more details, we refer the reader to $[4,7-9,20,24-26,37-48]$.

○2014 Imdad et al.: licensee Springer. This is an Open Access article distributed under the terms of the Creative Commons Attribution License (http://creativecommons.org/licenses/by/2.0), which permits unrestricted use, distribution, and reproduction in any medium, provided the original work is properly cited. 
Samet [27] introduced the concept of generalized Meir-Keeler type contraction function and proved some coupled fixed point theorems in partially ordered metric spaces. In different years, many researchers studied and worked on this contraction condition. Recently, in [12], Berinde and Pǎcurar gave the concept of symmetric Meir-Keeler type condition and generalized several results in the literature. Very recently, Erduran and Imdad [49] generalized the coupled fixed point theorems in the context of partial metric spaces. In this paper, we established some $n$-tupled fixed point theorems for generalized MeirKeeler type contraction condition in ordered partial metric spaces enjoying strict mixed monotone property. In this paper, we prove the existence and uniqueness of some MeirKeeler type $n$-tupled fixed point theorems in the context of partially ordered partial metric spaces. The presented theorems extend and improve the recent coupled fixed point theorems due to Erduran and Imdad [49].

\section{Preliminaries}

In this section, we collect some definitions and properties of partial metric space which are relevant to our presentation.

Definition 2.1 A partial metric on a nonempty set $X$ is a function $p: X \times X \rightarrow \mathbb{R}^{+}$such that for all $x, y, z \in X$,

(p1) $x=y \Leftrightarrow p(x, x)=p(x, y)=p(y, y)$,

(p2) $p(x, x) \leq p(x, y)$,

(p3) $p(x, y)=p(y, x)$

(p4) $p(x, y) \leq p(x, z)+p(z, y)-p(z, z)$.

A partial metric space is a pair $(X, p)$ such that $X$ is a nonempty set and $p$ is a partial metric on $X$.

Remark 2.1 It is clear that if $p(x, y)=0$, then from (p1), (p2) and (p3), $x=y$. But if $x=y$, $p(x, y)$ may not be zero.

Each partial metric $p$ on $X$ generates a $T_{0}$ topology $\tau_{p}$ on $X$ which has as a base the family of open $p$-balls $\left\{B_{p}(x, \epsilon), x \in X, \epsilon>0\right\}$, where $B_{p}(x, \epsilon)=\{y \in X: p(x, y)<p(x, x)+\epsilon\}$ for all $x \in X$ and $\epsilon>0$.

If $p$ is a partial metric on $X$, then the function $p^{s}: X \times X \rightarrow \mathbb{R}^{+}$given by

$$
p^{s}(x, y)=2 p(x, y)-p(x, x)-p(y, y)
$$

is a metric on $X$.

Example 2.1 $[36,37,43]$ Consider $X=\mathbb{R}^{+}$with $p(x, y)=\max \{x, y\}$. Then $\left(\mathbb{R}^{+}, p\right)$ is a partial metric space. It is clear that $p$ is not a (usual) metric. Note that in this case $p^{s}(x, y)=|x-y|$.

Example 2.2 [50] Let $X=\{[a, b]: a, b \in \mathbb{R}, a \leq b\}$ and define $p([a, b],[c, d])=\max \{b, d\}-$ $\min \{a, c\}$. Then $(X, p)$ is a partial metric space.

Example 2.3 [50] Let $X=[0,1] \cup[2,3]$ and define $p: X \times X \rightarrow[0, \infty)$ by

$$
p(x, y)= \begin{cases}\max \{x, y\}, & \{x, y\} \cap[2,3] \neq \emptyset ; \\ |x-y|, & \{x, y\} \subset[0,1] .\end{cases}
$$


Then $(X, p)$ is a complete partial metric space.

Example 2.4 [51] Let $(X, d)$ and $(X, p)$ be metric space and partial metric space, respectively. Then the mappings $\rho_{i}: X \times X \rightarrow \mathbb{R}^{+}(i \in\{1,2,3\})$ defined by

$$
\begin{aligned}
& \rho_{1}(x, y)=d(x, y)+p(x, y), \\
& \rho_{2}(x, y)=d(x, y)+\max \{\omega(x), \omega(y)\}, \\
& \rho_{3}(x, y)=d(x, y)+a
\end{aligned}
$$

induce partial metrics on $X$, where $\omega: X \rightarrow \mathbb{R}^{+}$is an arbitrary function and $a \geq 0$.

Definition 2.2 Let $(X, p)$ be a partial metric space and $\left\{x_{n}\right\}$ be a sequence in $X$. Then

(i) $\left\{x_{n}\right\}$ converges to a point $x \in X$ if and only if $p(x, x)=\lim _{n \rightarrow+\infty} p\left(x, x_{n}\right)$,

(ii) $\left\{x_{n}\right\}$ is said to be a Cauchy sequence if $\lim _{n, m \rightarrow+\infty} p\left(x_{n}, x_{m}\right)$ exists (and is finite).

Definition 2.3 A partial metric space $(X, p)$ is said to be complete if every Cauchy sequence $\left\{x_{n}\right\} \in X$ converges with respect to $\tau_{p}$, to a point $x \in X$, such that $p(x, x)=$ $\lim _{n, m \rightarrow+\infty} p\left(x_{n}, x_{m}\right)$.

Lemma 2.1 Let $(X, p)$ be a partial metric space. Then

(i) $\left\{x_{n}\right\}$ is a Cauchy sequence in $(X, p)$ if and only if it is a Cauchy sequence in the metric space $\left(X, p^{s}\right)$,

(ii) $(X, p)$ is complete if and only if the metric space $\left(X, p^{s}\right)$ is complete. Furthermore, $\lim _{n \rightarrow+\infty} p^{s}\left(x_{n}, x\right)=0$ if and only if

$$
p(x, x)=\lim _{n \rightarrow+\infty} p\left(x_{n}, x\right)=\lim _{n, m \rightarrow+\infty} p\left(x_{n}, x_{m}\right)
$$

In [52], Meir-Keeler generalized the well-known Banach fixed point theorem by proving the following interesting fixed point theorem.

Theorem 2.1 [52] Let $(X, d)$ be a complete metric space and $T: X \rightarrow X$ be a mapping. Suppose that for every $\epsilon>0$ there exists $\delta(\epsilon)>0$ such that for all

$$
x, y \in X \quad \text { with } \epsilon \leq d(x, y)<\epsilon+\delta(\epsilon) \quad \Rightarrow \quad d(T x, T y)<\epsilon .
$$

Then $T$ has a unique fixed point $z \in X$ and for all $x \in X$, the sequence $\left\{T^{n} x\right\}$ converges to $z$.

In recent years, many authors generalized Meir-Keeler fixed point theorems in various ways in various spaces which include complete metric space as well as ordered metric space. In [27], Samet introduced the concept of generalized Meir-Keeler type contraction function and proved some coupled fixed point results. Samet [27] introduced the definition below to modify the Meir-Keeler contraction and extended its applications.

Definition 2.4 [27] Let $(X, d)$ be a partially ordered metric space and $F: X \times X \rightarrow X$ be a given mapping. Then $F$ is a generalized Meir-Keeler type function if for all $\epsilon>0$ there 
exists $\delta(\epsilon)>0$ such that

$$
u \preceq x, y \preceq v, \quad \epsilon \leq \frac{1}{2}[d(x, u)+d(y, v)]<\epsilon+\delta(\epsilon) \quad \Rightarrow \quad d(F(x, y), F(u, v))<\epsilon .
$$

Very recently Erduran and Imdad [49] generalized the results of Samet [27] for ordered partial metric spaces. For more details, see [12, 27, 53, 54].

Erduran and Imdad [49] proved the following result:

Theorem 2.2 [49] Let $(X, \preceq)$ be a partially ordered set and suppose there is a partial metric $p$ on $X$ such that $(X, p)$ is complete partial metric space. Let $F: X \times X \rightarrow X$ be mapping satisfying the following hypotheses:

(1) F has the mixed strict monotone property,

(2) F is a generalized Meir-Keeler type function,

(3) $F$ is continuous or $X$ has the following properties:

(a) if a nondecreasing sequence $\left\{x_{n}\right\} \rightarrow x$, then $x_{n} \preceq x$ for all $n$,

(b) if a nonincreasing sequence $\left\{x_{n}\right\} \rightarrow x$, then $x \preceq x_{n}$ for all $n$.

If there exist $x_{0}, y_{0} \in X$ such that $x_{0} \prec F\left(x_{0}, y_{0}\right)$ and $F\left(y_{0}, x_{0}\right) \preceq y_{0}$, then there exists $(x, y) \in$ $X \times X$ such that $x=F(x, y)$ and $y=F(y, x)$. Furthermore, $p(x, x)=p(y, y)=0$.

Note Throughout the paper we consider $n$ to be an even integer.

Let $(X, p)$ be a partial metric. We endow $X \times X \times \cdots \times X, n$ times $\left(=X^{n}\right)$ with the partial metric $\eta$ defined for $\left(x^{1}, x^{2}, \ldots, x^{n}\right),\left(y^{1}, y^{2}, \ldots, y^{n}\right) \in X^{n}$ by

$$
\eta\left(\left(x^{1}, x^{2}, \ldots, x^{n}\right),\left(y^{1}, y^{2}, \ldots, y^{n}\right)\right)=\max \left\{p\left(x^{1}, y^{1}\right), p\left(x^{2}, y^{2}\right), \ldots, p\left(x^{n}, y^{n}\right)\right\}
$$

Let $F: X^{n} \rightarrow X$ be a given mapping. Then for all $\left(x^{1}, x^{2}, \ldots, x^{n}\right) \in X^{n}$ and for all $m \in \mathbb{N}$, $m \geq 2$, we denote

$$
\begin{aligned}
& F^{m}\left(x^{1}, x^{2}, \ldots, x^{n}\right) \\
& \quad=F\left(F^{m-1}\left(x^{1}, x^{2}, \ldots, x^{n}\right), F^{m-1}\left(x^{2}, \ldots, x^{n}, x^{1}\right), \ldots, F^{m-1}\left(x^{n}, x^{1}, \ldots, x^{n-1}\right)\right) .
\end{aligned}
$$

In this paper, we used the concept of $n$-tupled fixed point given by Samet and Vetro [34]. We recall some basic concepts.

Definition 2.5 [35] Let $(X, \preceq)$ be a partially ordered set and $F: X^{n} \rightarrow X$ be a mapping. The mapping $F$ is said to have the mixed monotone property if $F$ is nondecreasing in its odd position arguments and nonincreasing in its even position arguments, that is, if,

(i) for all $x_{1}^{1}, x_{2}^{1} \in X, \quad x_{1}^{1} \preceq x_{2}^{1} \Rightarrow F\left(x_{1}^{1}, x^{2}, x^{3}, \ldots, x^{n}\right) \preceq F\left(x_{2}^{1}, x^{2}, x^{3}, \ldots, x^{n}\right)$,

(ii) for all $x_{1}^{2}, x_{2}^{2} \in X, \quad x_{1}^{2} \preceq x_{2}^{2} \Rightarrow F\left(x^{1}, x_{2}^{2}, x^{3}, \ldots, x^{n}\right) \preceq F\left(x^{1}, x_{1}^{2}, x^{3}, \ldots, x^{n}\right)$,

(iii) for all $x_{1}^{3}, x_{2}^{3} \in X, \quad x_{1}^{3} \preceq x_{2}^{3} \Rightarrow F\left(x^{1}, x^{2}, x_{1}^{3}, \ldots, x^{n}\right) \preceq F\left(x^{1}, x^{2}, x_{2}^{3}, \ldots, x^{n}\right)$,

$$
\text { for all } x_{1}^{n}, x_{2}^{n} \in X, \quad x_{1}^{n} \preceq x_{2}^{n} \Rightarrow F\left(x^{1}, x^{2}, x^{3}, \ldots, x_{2}^{n}\right) \preceq F\left(x^{1}, x^{2}, x^{3}, \ldots, x_{1}^{n}\right) \text {. }
$$


Definition 2.6 [34] An element $\left(x^{1}, x^{2}, \ldots, x^{n}\right) \in X^{n}$ is called an $n$-tupled fixed point of the mapping $F: X^{n} \rightarrow X$ if

$$
\left\{\begin{array}{l}
F\left(x^{1}, x^{2}, x^{3}, \ldots, x^{n}\right)=x^{1}, \\
F\left(x^{2}, x^{3}, \ldots, x^{n}, x^{1}\right)=x^{2}, \\
F\left(x^{3}, \ldots, x^{n}, x^{1}, x^{2}\right)=x^{3}, \\
\vdots \\
F\left(x^{n}, x^{1}, x^{2}, \ldots, x^{n-1}\right)=x^{n} .
\end{array}\right.
$$

Example 2.5 Let $(R, d)$ be a partially ordered metric space under natural setting and let $F: R^{n} \rightarrow R$ be a mapping defined by $F\left(x^{1}, x^{2}, x^{3}, \ldots, x^{n}\right)=\sin \left(x^{1} \cdot x^{2} \cdot x^{3} \cdots x^{n}\right)$, for any $x^{1}, x^{2}, \ldots, x^{n} \in R$. Then $(0,0, \ldots, 0)$ is an $n$-tupled fixed point of $F$.

Remark 2.2 Definition 2.6 with $n=2,4$ respectively yields the definition of coupled fixed point [13] and quadrupled fixed point [55].

\section{Main results}

We begin this section by defining the following definitions:

Definition 3.1 Let $(X, \preceq)$ be a partially ordered set and $F: X^{n} \rightarrow X$ be a mapping. The mapping $F$ is said to have the mixed strict monotone property if $F$ is nondecreasing in its odd position arguments and nonincreasing in its even position arguments, that is, if,

(i) for all $x_{1}^{1}, x_{2}^{1} \in X, \quad x_{1}^{1} \prec x_{2}^{1} \Rightarrow F\left(x_{1}^{1}, x^{2}, x^{3}, \ldots, x^{n}\right) \prec F\left(x_{2}^{1}, x^{2}, x^{3}, \ldots, x^{n}\right)$,

(ii) for all $x_{1}^{2}, x_{2}^{2} \in X, \quad x_{1}^{2} \prec x_{2}^{2} \Rightarrow F\left(x^{1}, x_{2}^{2}, x^{3}, \ldots, x^{n}\right) \prec F\left(x^{1}, x_{1}^{2}, x^{3}, \ldots, x^{n}\right)$,

(iii) for all $x_{1}^{3}, x_{2}^{3} \in X, \quad x_{1}^{3} \prec x_{2}^{3} \Rightarrow F\left(x^{1}, x^{2}, x_{1}^{3}, \ldots, x^{n}\right) \prec F\left(x^{1}, x^{2}, x_{2}^{3}, \ldots, x^{n}\right)$,

$$
\text { for all } x_{1}^{n}, x_{2}^{n} \in X, \quad x_{1}^{n} \prec x_{2}^{n} \Rightarrow F\left(x^{1}, x^{2}, x^{3}, \ldots, x_{2}^{n}\right) \prec F\left(x^{1}, x^{2}, x^{3}, \ldots, x_{1}^{n}\right) \text {. }
$$

Definition 3.2 Let $(X, p)$ be a partially ordered partial metric space and $F: X^{n} \rightarrow X$ be a given mapping. We say that $F$ is a generalized Meir-Keeler type function if for all $\epsilon>$ 0 there exists $\delta(\epsilon)>0$ such that for $\left(x^{1}, x^{2}, \ldots, x^{n}\right),\left(y^{1}, y^{2}, \ldots, y^{n}\right) \in X^{n}$ with $x^{1} \preceq y^{1}, y^{2} \preceq$ $x^{2}, x^{3} \preceq y^{3}, \ldots, y^{n} \preceq x^{n}$

$$
\left\{\begin{array}{c}
\epsilon \leq \max \left\{p\left(x^{1}, y^{1}\right), p\left(x^{2}, y^{2}\right), p\left(x^{3}, y^{3}\right), \ldots, p\left(x^{n}, y^{n}\right)\right\}<\epsilon+\delta(\epsilon) \\
\Rightarrow p\left(F\left(x^{1}, x^{2}, x^{3}, \ldots, x^{n}\right), F\left(y^{1}, y^{2}, y^{3}, \ldots, y^{n}\right)\right)<\epsilon .
\end{array}\right.
$$

The aim of this work is to prove the following results:

Lemma 3.1 Let $(X, \preceq)$ be a partially ordered set and suppose that there is a partial metric $p$ on $X$ such that $(X, p)$ is a complete partial metric space. Let $F: X^{n} \rightarrow X$ be a given mapping. If $F$ is a generalized Meir-Keeler type function, then for $\left(x^{1}, x^{2}, \ldots, x^{n}\right),\left(y^{1}, y^{2}, \ldots, y^{n}\right) \in X^{n}$

$$
p\left(F\left(x^{1}, x^{2}, \ldots, x^{n}\right), F\left(y^{1}, y^{2}, \ldots, y^{n}\right)\right)<\max \left\{p\left(x^{1}, y^{1}\right), p\left(x^{2}, y^{2}\right), \ldots, p\left(x^{n}, y^{n}\right)\right\}
$$

with $x^{1} \prec y^{1}, y^{2} \preceq x^{2}, x^{3} \prec y^{3}, \ldots, y^{n} \preceq x^{n}$ or $x^{1} \preceq y^{1}, y^{2} \prec x^{2}, x^{3} \preceq y^{3}, \ldots, y^{n} \prec x^{n}$. 
Proof Let $x^{1}, x^{2}, \ldots, x^{n}, y^{1}, y^{2}, \ldots, y^{n} \in X$ such that

$$
\begin{array}{lllll}
x^{1} \prec y^{1}, & y^{2} \preceq x^{2}, & x^{3} \prec y^{3}, & \ldots, & y^{n} \preceq x^{n} \quad \text { or } \\
x^{1} \preceq y^{1}, & y^{2} \prec x^{2}, & x^{3} \preceq y^{3}, & \ldots, & y^{n} \prec x^{n} .
\end{array}
$$

Then $\max \left\{p\left(x^{1}, y^{1}\right), p\left(x^{2}, y^{2}\right), p\left(x^{3}, y^{3}\right), \ldots, p\left(x^{n}, y^{n}\right)\right\}>0$. Since $F$ is a generalized MeirKeeler type function. Therefore for $\epsilon=\max \left\{p\left(x^{1}, y^{1}\right), p\left(x^{2}, y^{2}\right), \ldots, p\left(x^{n}, y^{n}\right)\right\}$, there exists $\delta(\epsilon)>0$ such that

$$
\begin{aligned}
x_{*}^{1} \preceq y_{*}^{1}, \quad y_{*}^{2} \preceq x_{*}^{2}, \quad x_{*}^{3} \preceq y_{*}^{3}, \quad \ldots, \quad y_{*}^{n} \preceq x_{*}^{n}, \\
\epsilon \leq \max \left\{p\left(x_{*}^{1}, y_{*}^{1}\right), p\left(x_{*}^{2}, y_{*}^{2}\right), p\left(x_{*}^{3}, y_{*}^{3}\right), \ldots, p\left(x_{*}^{n}, y_{*}^{n}\right)\right\}<\epsilon+\delta(\epsilon) \\
\Rightarrow \quad p\left(F\left(x_{*}^{1}, x_{*}^{2}, x_{*}^{3}, \ldots, x_{*}^{n}\right), F\left(y_{*}^{1}, y_{*}^{2}, y_{*}^{3}, \ldots, y_{*}^{n}\right)\right)<\epsilon .
\end{aligned}
$$

Putting $x_{*}^{1}=x^{1}, x_{*}^{2}=x^{2}, \ldots, x_{*}^{n}=x^{n}$ and $y_{*}^{1}=y^{1}, y_{*}^{2}=y^{2}, \ldots, y_{*}^{n}=y^{n}$, we obtain the desired result.

Lemma 3.2 Let $(X, \preceq)$ be a partially ordered set and suppose that there is a partial metric $p$ on $X$ such that $(X, p)$ is a complete partial metric space. Let $F: X^{n} \rightarrow X$ be a given mapping. Assume that the following hypotheses hold:

(1) F has the mixed strict monotone property,

(2) $F$ is a generalized Meir-Keeler type function,

(3) there exist $\left(x^{1}, x^{2}, \ldots, x^{n}\right),\left(y^{1}, y^{2}, \ldots, y^{n}\right) \in X^{n}$ with $x^{1} \prec y^{1}, y^{2} \preceq x^{2}, x^{3} \prec y^{3}, \ldots, y^{n} \preceq x^{n}$.

Then

$$
\begin{aligned}
\eta( & \left(F^{m}\left(x^{1}, x^{2}, x^{3}, \ldots, x^{n}\right), F^{m}\left(x^{2}, x^{3}, \ldots, x^{n}, x^{1}\right), \ldots, F^{m}\left(x^{n}, x^{1}, x^{2}, \ldots, x^{n-1}\right)\right), \\
& \left.\left(F^{m}\left(y^{1}, y^{2}, y^{3}, \ldots, y^{n}\right), F^{m}\left(y^{2}, y^{3}, \ldots, y^{n}, y^{1}\right), \ldots, F^{m}\left(y^{n}, y^{1}, y^{2}, \ldots, y^{n-1}\right)\right)\right) \\
\rightarrow & 0 \quad \text { as } m \rightarrow \infty .
\end{aligned}
$$

Proof We claim that:

$$
\left\{\begin{array}{l}
F^{m}\left(x^{1}, x^{2}, x^{3}, \ldots, x^{n}\right) \prec F^{m}\left(y^{1}, y^{2}, y^{3}, \ldots, y^{n}\right), \\
F^{m}\left(y^{2}, y^{3}, \ldots, y^{n}, y^{1}\right) \prec F^{m}\left(x^{2}, x^{3}, \ldots, x^{n}, x^{1}\right), \\
\vdots \\
F^{m}\left(y^{n}, y^{1}, y^{2}, \ldots, y^{n-1}\right) \prec F^{m}\left(x^{n}, x^{1}, x^{2}, \ldots, x^{n-1}\right),
\end{array}\right.
$$

with the notation $F^{1} \equiv F$. Then by the mixed strict monotone property of $F$,

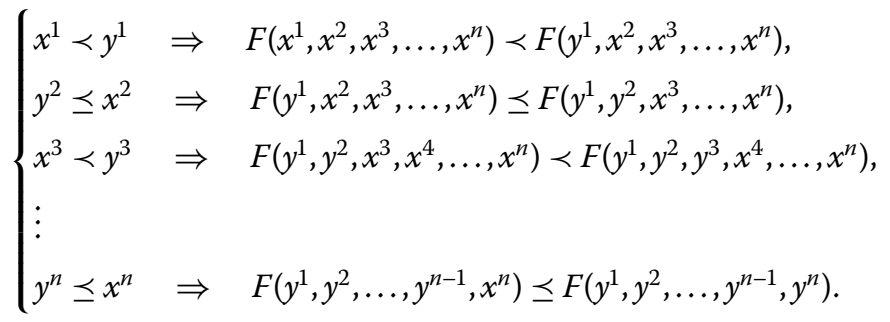


Then we have $F\left(x^{1}, x^{2}, x^{3}, \ldots, x^{n}\right) \prec F\left(y^{1}, y^{2}, y^{3}, \ldots, y^{n}\right)$. Also

$$
\left\{\begin{array}{l}
y^{2} \preceq x^{2} \quad \Rightarrow \quad F\left(y^{2}, y^{3}, \ldots, y^{n}, y^{1}\right) \prec F\left(x^{2}, y^{3}, \ldots, y^{n}, y^{1}\right), \\
x^{3} \prec y^{3} \quad \Rightarrow \quad F\left(x^{2}, y^{3}, y^{4}, \ldots, y^{n}, y^{1}\right) \preceq F\left(x^{2}, x^{3}, y^{4}, \ldots, y^{n}, y^{1}\right), \\
\vdots \\
y^{n} \preceq x^{n} \quad \Rightarrow \quad F\left(x^{2}, x^{3}, \ldots, y^{n}, y^{1}\right) \prec F\left(x^{2}, x^{3}, \ldots, x^{n}, y^{1}\right), \\
x^{1} \prec y^{1} \quad \Rightarrow \quad F\left(x^{2}, x^{3}, \ldots, x^{n}, y^{1}\right) \preceq F\left(x^{2}, x^{3}, \ldots, x^{n}, x^{1}\right) .
\end{array}\right.
$$

Therefore $F\left(y^{2}, y^{3}, \ldots, y^{n}, y^{1}\right) \prec F\left(x^{2}, x^{3}, \ldots, x^{n}, x^{1}\right)$.

And similarly

$$
\left\{\begin{array}{l}
y^{n} \preceq x^{n} \quad \Rightarrow \quad F\left(y^{n}, y^{1}, y^{2}, \ldots, y^{n-1}\right) \prec F\left(x^{n}, y^{1}, y^{2}, \ldots, y^{n-1}\right), \\
x^{1} \prec y^{1} \quad \Rightarrow \quad F\left(x^{n}, y^{1}, y^{2}, \ldots, y^{n-1}\right) \preceq F\left(x^{n}, x^{1}, y^{2}, \ldots, y^{n-1}\right), \\
y^{2} \preceq x^{2} \quad \Rightarrow \quad F\left(x^{n}, x^{1}, y^{2}, \ldots, y^{n-1}\right) \prec F\left(x^{n}, x^{1}, x^{2}, y^{3}, \ldots, y^{n-1}\right), \\
\vdots \\
x^{n-1} \prec y^{n-1} \quad
\end{array}\right.
$$

Therefore $F\left(y^{n}, y^{1}, y^{2}, \ldots, y^{n-1}\right) \prec F\left(x^{n}, x^{1}, x^{2}, \ldots, x^{n-1}\right)$. Thus (3.3) is satisfied for $m=1$. For $m=2$, we use the same strategy. We have

$$
\begin{aligned}
& F\left(x^{1}, x^{2}, x^{3}, \ldots, x^{n}\right) \prec F\left(y^{1}, y^{2}, y^{3}, \ldots, y^{n}\right) \\
& \Rightarrow \quad F\left(F\left(x^{1}, x^{2}, x^{3}, \ldots, x^{n}\right), F\left(x^{2}, x^{3}, \ldots, x^{n}, x^{1}\right), \ldots, F\left(x^{n}, x^{1}, x^{2}, \ldots, x^{n-1}\right)\right) \\
& \prec F\left(F\left(y^{1}, y^{2}, y^{3}, \ldots, y^{n}\right), F\left(x^{2}, x^{3}, \ldots, x^{n}, x^{1}\right), \ldots, F\left(x^{n}, x^{1}, x^{2}, \ldots, x^{n-1}\right)\right), \\
& F\left(y^{2}, y^{3}, \ldots, y^{n}, y^{1}\right) \prec F\left(x^{2}, x^{3}, \ldots, x^{n}, x^{1}\right) \\
& \Rightarrow \quad F\left(F\left(y^{1}, y^{2}, y^{3}, \ldots, y^{n}\right), F\left(x^{2}, x^{3}, \ldots, x^{n}, x^{1}\right), \ldots, F\left(x^{n}, x^{1}, x^{2}, \ldots, x^{n-1}\right)\right) \\
& \prec F\left(F\left(y^{1}, y^{2}, y^{3}, \ldots, y^{n}\right), F\left(y^{2}, y^{3}, \ldots, y^{n}, y^{1}\right), \ldots, F\left(x^{n}, x^{1}, x^{2}, \ldots, x^{n-1}\right)\right), \\
& \vdots \\
& F\left(y^{n}, y^{1}, y^{2}, \ldots, y^{n-1}\right) \prec F\left(x^{n}, y^{1}, y^{2}, \ldots, y^{n-1}\right) \\
& \Rightarrow \quad F\left(F\left(y^{1}, y^{2}, y^{3}, \ldots, y^{n}\right), F\left(y^{2}, y^{3}, \ldots, y^{n}, y^{1}\right), \ldots, F\left(x^{n}, x^{1}, x^{2}, \ldots, x^{n-1}\right)\right) \\
& \prec F\left(F\left(y^{1}, y^{2}, y^{3}, \ldots, y^{n}\right), F\left(y^{2}, y^{3}, \ldots, y^{n}, y^{1}\right), \ldots, F\left(y^{n}, y^{1}, y^{2}, \ldots, y^{n-1}\right)\right) .
\end{aligned}
$$

Thus we get

$$
F^{2}\left(x^{1}, x^{2}, x^{3}, \ldots, x^{n}\right) \prec F^{2}\left(y^{1}, y^{2}, y^{3}, \ldots, y^{n}\right) .
$$

Now,

$$
\begin{aligned}
& F\left(y^{2}, y^{3}, \ldots, y^{n}, y^{1}\right) \prec F\left(x^{2}, x^{3}, \ldots, x^{n}, x^{1}\right) \\
& \quad \Rightarrow \quad F\left(F\left(y^{2}, y^{3}, \ldots, y^{n}, y^{1}\right), \ldots, F\left(y^{n}, y^{1}, y^{2}, \ldots, y^{n-1}\right), F\left(y^{1}, y^{2}, y^{3}, \ldots, y^{n}\right)\right)
\end{aligned}
$$




$$
\prec F\left(F\left(x^{2}, x^{3}, \ldots, x^{n}, x^{1}\right), \ldots, F\left(y^{n}, y^{1}, y^{2}, \ldots, y^{n-1}\right), F\left(y^{1}, y^{2}, y^{3}, \ldots, y^{n}\right)\right)
$$

$\vdots$

$$
\begin{aligned}
& F\left(y^{n}, y^{1}, y^{2}, \ldots, y^{n-1}\right) \prec F\left(x^{n}, x^{1}, x^{2}, \ldots, x^{n-1}\right) \\
& \Rightarrow \quad F\left(F\left(x^{2}, x^{3}, \ldots, x^{n}, x^{1}\right), F\left(y^{n}, y^{1}, y^{2}, \ldots, y^{n-1}\right), \ldots, F\left(y^{1}, y^{2}, y^{3}, \ldots, y^{n}\right)\right) \\
& \quad \prec F\left(F\left(x^{2}, x^{3}, \ldots, x^{n}, x^{1}\right), F\left(x^{n}, x^{1}, x^{2}, \ldots, x^{n-1}\right), \ldots, F\left(y^{1}, y^{2}, y^{3}, \ldots, y^{n}\right)\right), \\
& F\left(x^{1}, x^{2}, x^{3}, \ldots, x^{n}\right) \prec F\left(y^{1}, y^{2}, y^{3}, \ldots, y^{n}\right) \\
& \Rightarrow \quad F\left(F\left(x^{2}, x^{3}, \ldots, x^{n}, x^{1}\right), \ldots, F\left(x^{n}, x^{1}, x^{2}, \ldots, x^{n-1}\right), F\left(y^{1}, y^{2}, y^{3}, \ldots, y^{n}\right)\right) \\
& \quad \prec F\left(F\left(x^{2}, x^{3}, \ldots, x^{n}, x^{1}\right), \ldots, F\left(x^{n}, x^{1}, x^{2}, \ldots, x^{n-1}\right), F\left(x^{1}, x^{2}, x^{3}, \ldots, x^{n}\right)\right) .
\end{aligned}
$$

Therefore we get

$$
F^{2}\left(y^{2}, y^{3}, \ldots, y^{n}, y^{1}\right) \prec F^{2}\left(x^{2}, x^{3}, \ldots, x^{n}, x^{1}\right) .
$$

In the same way,

$$
\begin{aligned}
& F\left(y^{n}, y^{1}, y^{2}, \ldots, y^{n-1}\right) \prec F\left(x^{n}, x^{1}, x^{2}, \ldots, x^{n-1}\right) \\
& \Rightarrow \quad F\left(F\left(y^{n}, y^{1}, y^{2}, \ldots, y^{n-1}\right), F\left(y^{1}, y^{2}, y^{3}, \ldots, y^{n}\right), \ldots, F\left(y^{n-1}, \ldots, y^{2}, y^{1}, y^{n}\right)\right) \\
& \prec F\left(F\left(x^{n}, x^{1}, x^{2}, \ldots, x^{n-1}\right), F\left(y^{1}, y^{2}, y^{3}, \ldots, y^{n}\right), \ldots, F\left(y^{n-1}, \ldots, y^{2}, y^{1}, y^{n}\right)\right), \\
& \vdots \\
& F\left(x^{1}, x^{2}, x^{3}, \ldots, x^{n}\right) \prec F\left(y^{1}, y^{2}, y^{3}, \ldots, y^{n}\right) \\
& \Rightarrow \quad F\left(F\left(x^{n}, x^{1}, x^{2}, \ldots, x^{n-1}\right), F\left(y^{1}, y^{2}, y^{3}, \ldots, y^{n}\right), \ldots, F\left(x^{n-1}, \ldots, x^{2}, x^{1}, x^{n}\right)\right) \\
& \prec F\left(F\left(x^{n}, x^{1}, x^{2}, \ldots, x^{n-1}\right), F\left(x^{1}, x^{2}, x^{3}, \ldots, x^{n}\right), \ldots, F\left(x^{n-1}, \ldots, x^{2}, x^{1}, x^{n}\right)\right), \\
& F\left(y^{2}, y^{3}, \ldots, y^{n}, y^{1}\right) \prec F\left(x^{2}, x^{3}, \ldots, x^{n}, x^{1}\right) \\
& \Rightarrow \quad F\left(F\left(x^{n}, x^{1}, \ldots, x^{n-1}\right), F\left(x^{1}, x^{2}, \ldots, x^{n}\right), F\left(y^{2}, \ldots, y^{n}, y^{1}\right), \ldots, F\left(x^{n-1}, \ldots, x^{1}, x^{n}\right)\right) \\
& \prec F\left(F\left(x^{n}, x^{1}, \ldots, x^{n-1}\right), F\left(x^{1}, x^{2}, \ldots, x^{n}\right),\right. \\
& \left.F\left(x^{2}, \ldots, x^{n}, x^{1}\right), \ldots, F\left(x^{n-1}, \ldots, x^{1}, x^{n}\right)\right) \text {. }
\end{aligned}
$$

Thus we have

$$
F^{2}\left(y^{n}, y^{1}, y^{2}, \ldots, y^{n-1}\right) \prec F^{2}\left(x^{n}, x^{1}, x^{2}, \ldots, x^{n-1}\right)
$$

Thus (3.3) is satisfied for $m=2$. Repeating the same argument for each $m$, we see that (3.3) holds. Now using Lemma 3.1 and (3.3), we get

$$
\begin{aligned}
p\left(F^{m+1}\left(x^{1}, x^{2}, x^{3}, \ldots, x^{n}\right), F^{m+1}\left(y^{1}, y^{2}, y^{3}, \ldots, y^{n}\right)\right) & \\
= & p\left(F\left(F^{m}\left(x^{1}, x^{2}, x^{3}, \ldots, x^{n}\right), F^{m}\left(x^{2}, x^{3}, \ldots, x^{n}, x^{1}\right), \ldots, F^{m}\left(x^{n}, x^{1}, x^{2}, \ldots, x^{n-1}\right)\right),\right. \\
& \left.F\left(F^{m}\left(y^{1}, y^{2}, y^{3}, \ldots, y^{n}\right), F^{m}\left(y^{2}, y^{3}, \ldots, y^{n}, y^{1}\right), \ldots, F^{m}\left(y^{n}, y^{1}, y^{2}, \ldots, y^{n-1}\right)\right)\right)
\end{aligned}
$$




$$
\begin{gathered}
<\max \left[p\left(F^{m}\left(x^{1}, x^{2}, x^{3}, \ldots, x^{n}\right), F^{m}\left(y^{1}, y^{2}, y^{3}, \ldots, y^{n}\right)\right),\right. \\
p\left(F^{m}\left(x^{2}, x^{3}, \ldots, x^{n}, x^{1}\right), F^{m}\left(y^{2}, y^{3}, \ldots, y^{n}, y^{1}\right)\right), \ldots, \\
\left.p\left(F^{m}\left(x^{n}, x^{1}, x^{2}, \ldots, x^{n-1}\right), F^{m}\left(y^{n}, y^{1}, y^{2}, \ldots, y^{n-1}\right)\right)\right] .
\end{gathered}
$$

Also we have

$$
\begin{aligned}
p( & \left.F^{m+1}\left(x^{2}, x^{3}, \ldots, x^{n}, x^{1}\right), F^{m+1}\left(y^{2}, y^{3}, \ldots, y^{n}, y^{1}\right)\right) \\
= & p\left(F\left(F^{m}\left(x^{2}, x^{3}, \ldots, x^{n}, x^{1}\right), \ldots, F^{m}\left(x^{n}, x^{1}, x^{2}, \ldots, x^{n-1}\right), F^{m}\left(x^{1}, x^{2}, x^{3}, \ldots, x^{n}\right)\right),\right. \\
& \left.F\left(F^{m}\left(y^{2}, y^{3}, \ldots, y^{n}, y^{1}\right), \ldots, F^{m}\left(y^{n}, y^{1}, y^{2}, \ldots, y^{n-1}\right), F^{m}\left(y^{1}, y^{2}, y^{3}, \ldots, y^{n}\right)\right)\right) \\
< & \max \left[p\left(F^{m}\left(x^{2}, x^{3}, \ldots, x^{n}, x^{1}\right), F^{m}\left(y^{2}, y^{3}, \ldots, y^{n}, y^{1}\right)\right), \ldots, p\left(F^{m}\left(x^{n}, x^{1}, x^{2}, \ldots, x^{n-1}\right),\right.\right. \\
& \left.\left.F^{m}\left(y^{n}, y^{1}, y^{2}, \ldots, y^{n-1}\right)\right), p\left(F^{m}\left(x^{1}, x^{2}, x^{3}, \ldots, x^{n}\right), F^{m}\left(y^{1}, y^{2}, y^{3}, \ldots, y^{n}\right)\right)\right] .
\end{aligned}
$$

Similarly we have,

$$
\begin{aligned}
& p\left(F^{m+1}\left(x^{n}, x^{1}, x^{2}, \ldots, x^{n-1}\right), F^{m+1}\left(y^{n}, y^{1}, y^{2}, \ldots, y^{n-1}\right)\right) \\
&< \max \left[p\left(F^{m}\left(x^{n}, x^{1}, x^{2}, \ldots, x^{n-1}\right), F^{m}\left(y^{n}, y^{1}, y^{2}, \ldots, y^{n-1}\right)\right),\right. \\
& p\left(F^{m}\left(x^{1}, x^{2}, x^{3}, \ldots, x^{n}\right), F^{m}\left(y^{1}, y^{2}, y^{3}, \ldots, y^{n}\right)\right), \ldots, \\
&\left.p\left(F^{m}\left(x^{n-1}, \ldots, x^{2}, x^{1}, x^{n}\right), F^{m}\left(y^{n-1}, \ldots, y^{2}, y^{1}, y^{n}\right)\right)\right] .
\end{aligned}
$$

Combining (3.4), (3.5), and (3.6), we get

$$
\begin{gathered}
\eta\left(\left(F^{m+1}\left(x^{1}, x^{2}, x^{3}, \ldots, x^{n}\right), F^{m+1}\left(x^{2}, x^{3}, \ldots, x^{n}, x^{1}\right), \ldots, F^{m+1}\left(x^{n}, x^{1}, x^{2}, \ldots, x^{n-1}\right)\right),\right. \\
\left.\left(F^{m+1}\left(y^{1}, y^{2}, y^{3}, \ldots, y^{n}\right), F^{m+1}\left(y^{2}, y^{3}, \ldots, y^{n}, y^{1}\right), \ldots, F^{m+1}\left(y^{n}, y^{1}, y^{2}, \ldots, y^{n-1}\right)\right)\right) \\
<\eta\left(\left(F^{m}\left(x^{1}, x^{2}, x^{3}, \ldots, x^{n}\right), F^{m}\left(x^{2}, x^{3}, \ldots, x^{n}, x^{1}\right), \ldots, F^{m}\left(x^{n}, x^{1}, x^{2}, \ldots, x^{n-1}\right)\right),\right. \\
\left.\left(F^{m}\left(y^{1}, y^{2}, y^{3}, \ldots, y^{n}\right), F^{m}\left(y^{2}, y^{3}, \ldots, y^{n}, y^{1}\right), \ldots, F^{m}\left(y^{n}, y^{1}, y^{2}, \ldots, y^{n-1}\right)\right)\right) .
\end{gathered}
$$

This implies that

$$
\begin{gathered}
\left\{\eta \left(\left(F^{m}\left(x^{1}, x^{2}, x^{3}, \ldots, x^{n}\right), F^{m}\left(x^{2}, x^{3}, \ldots, x^{n}, x^{1}\right), \ldots, F^{m}\left(x^{n}, x^{1}, x^{2}, \ldots, x^{n-1}\right)\right),\right.\right. \\
\left.\left.\left(F^{m}\left(y^{1}, y^{2}, y^{3}, \ldots, y^{n}\right), F^{m}\left(y^{2}, y^{3}, \ldots, y^{n}, y^{1}\right), \ldots, F^{m}\left(y^{n}, y^{1}, y^{2}, \ldots, y^{n-1}\right)\right)\right)\right\}
\end{gathered}
$$

is a decreasing convergent sequence. Thus there exists $\epsilon \geq 0$ such that

$$
\begin{gathered}
\lim _{m \rightarrow \infty}\left[\eta \left(\left(F^{m}\left(x^{1}, x^{2}, x^{3}, \ldots, x^{n}\right), F^{m}\left(x^{2}, x^{3}, \ldots, x^{n}, x^{1}\right), \ldots, F^{m}\left(x^{n}, x^{1}, x^{2}, \ldots, x^{n-1}\right)\right),\right.\right. \\
\left.\left.\left(F^{m}\left(y^{1}, y^{2}, y^{3}, \ldots, y^{n}\right), F^{m}\left(y^{2}, y^{3}, \ldots, y^{n}, y^{1}\right), \ldots, F^{m}\left(y^{n}, y^{1}, y^{2}, \ldots, y^{n-1}\right)\right)\right)\right]=\epsilon .
\end{gathered}
$$

Now we show that $\epsilon=0$. Assume that $\epsilon>0$. This implies that there exists $m_{0} \in \mathbb{N}$ such that

$$
\begin{aligned}
\epsilon< & \eta\left(\left(F^{m_{0}}\left(x^{1}, x^{2}, x^{3}, \ldots, x^{n}\right), F^{m_{0}}\left(x^{2}, x^{3}, \ldots, x^{n}, x^{1}\right), \ldots, F^{m_{0}}\left(x^{n}, x^{1}, x^{2}, \ldots, x^{n-1}\right)\right),\right. \\
& \left.\left(F^{m_{0}}\left(y^{1}, y^{2}, y^{3}, \ldots, y^{n}\right), F^{m_{0}}\left(y^{2}, y^{3}, \ldots, y^{n}, y^{1}\right), \ldots, F^{m_{0}}\left(y^{n}, y^{1}, y^{2}, \ldots, y^{n-1}\right)\right)\right) \\
< & \epsilon+\delta(\epsilon) .
\end{aligned}
$$


In this case we have

$$
\begin{aligned}
\epsilon \leq & \max \left\{p\left(F^{m_{0}}\left(x^{1}, x^{2}, x^{3}, \ldots, x^{n}\right), F^{m_{0}}\left(y^{1}, y^{2}, y^{3}, \ldots, y^{n}\right)\right), p\left(F^{m_{0}}\left(x^{2}, x^{3}, \ldots, x^{n}, x^{1}\right),\right.\right. \\
& \left.\left.F^{m_{0}}\left(y^{2}, y^{3}, \ldots, y^{n}, y^{1}\right)\right), \ldots, p\left(F^{m_{0}}\left(x^{n}, x^{1}, x^{2}, \ldots, x^{n-1}\right), F^{m_{0}}\left(y^{n}, y^{1}, y^{2}, \ldots, y^{n-1}\right)\right)\right\} \\
< & \epsilon+\delta(\epsilon) .
\end{aligned}
$$

It follows from (3.3) and hypothesis (2) that

$$
\begin{aligned}
& p\left(\left(F\left(F^{m_{0}}\left(x^{1}, x^{2}, x^{3}, \ldots, x^{n}\right), F^{m_{0}}\left(x^{2}, x^{3}, \ldots, x^{n}, x^{1}\right), \ldots, F^{m_{0}}\left(x^{n}, x^{1}, x^{2}, \ldots, x^{n-1}\right)\right)\right),\right. \\
& \left.\quad\left(F\left(F^{m_{0}}\left(y^{1}, y^{2}, y^{3}, \ldots, y^{n}\right), F^{m_{0}}\left(y^{2}, y^{3}, \ldots, y^{n}, y^{1}\right), \ldots, F^{m_{0}}\left(y^{n}, y^{1}, y^{2}, \ldots, y^{n-1}\right)\right)\right)\right)<\epsilon,
\end{aligned}
$$

that is,

$$
p\left(F^{m_{0}+1}\left(x^{1}, x^{2}, x^{3}, \ldots, x^{n}\right), F^{m_{0}+1}\left(y^{1}, y^{2}, y^{3}, \ldots, y^{n}\right)\right)<\epsilon
$$

On the other hand, we have

$$
\begin{aligned}
\epsilon \leq & \max \left\{p\left(F^{m_{0}}\left(x^{2}, x^{3}, \ldots, x^{n}, x^{1}\right), F^{m_{0}}\left(y^{2}, y^{3}, \ldots, y^{n}, y^{1}\right)\right), \ldots, p\left(F^{m_{0}}\left(x^{n}, x^{1}, x^{2}, \ldots, x^{n-1}\right),\right.\right. \\
& \left.\left.F^{m_{0}}\left(y^{n}, y^{1}, y^{2}, \ldots, y^{n-1}\right)\right), p\left(F^{m_{0}}\left(x^{1}, x^{2}, x^{3}, \ldots, x^{n}\right), F^{m_{0}}\left(y^{1}, y^{2}, y^{3}, \ldots, y^{n}\right)\right)\right\} \\
< & \epsilon+\delta(\epsilon),
\end{aligned}
$$

which implies that

$$
p\left(F^{m_{0}+1}\left(x^{2}, x^{3}, \ldots, x^{n}, x^{1}\right), F^{m_{0}+1}\left(y^{2}, y^{3}, \ldots, y^{n}, y^{1}\right)\right)<\epsilon
$$

Similarly,

$$
p\left(F^{m_{0}+1}\left(x^{n}, x^{1}, x^{2}, \ldots, x^{n-1}\right), F^{m_{0}+1}\left(y^{n}, y^{1}, y^{2}, \ldots, y^{n-1}\right)\right)<\epsilon .
$$

Combining (3.7), (3.8), and (3.9), we have

$$
\begin{aligned}
& \eta\left(\left(F^{m_{0}+1}\left(x^{1}, x^{2}, x^{3}, \ldots, x^{n}\right), F^{m_{0}+1}\left(x^{2}, x^{3}, \ldots, x^{n}, x^{1}\right), \ldots, F^{m_{0}+1}\left(x^{n}, x^{1}, x^{2}, \ldots, x^{n-1}\right)\right),\right. \\
& \left.\quad\left(F^{m_{0}+1}\left(y^{1}, y^{2}, y^{3}, \ldots, y^{n}\right), F^{m_{0}+1}\left(y^{2}, y^{3}, \ldots, y^{n}, y^{1}\right), \ldots, F^{m_{0}+1}\left(y^{n}, y^{1}, y^{2}, \ldots, y^{n-1}\right)\right)\right) \\
& \quad<\epsilon
\end{aligned}
$$

which is a contradiction. Therefore, we have necessarily $\epsilon=0$. That is,

$$
\begin{aligned}
& \eta\left(\left(F^{m}\left(x^{1}, x^{2}, x^{3}, \ldots, x^{n}\right), F^{m}\left(x^{2}, x^{3}, \ldots, x^{n}, x^{1}\right), \ldots, F^{m}\left(x^{n}, x^{1}, x^{2}, \ldots, x^{n-1}\right)\right),\right. \\
& \left.\quad\left(F^{m}\left(y^{1}, y^{2}, y^{3}, \ldots, y^{n}\right), F^{m}\left(y^{2}, y^{3}, \ldots, y^{n}, y^{1}\right), \ldots, F^{m}\left(y^{n}, y^{1}, y^{2}, \ldots, y^{n-1}\right)\right)\right)=0 .
\end{aligned}
$$

Remark 3.1 Lemma 3.2 also holds if we replace condition (3) by $\exists\left(x^{1}, x^{2}, x^{3}, \ldots, x^{n}\right),\left(y^{1}, y^{2}\right.$, $\left.y^{3}, \ldots, y^{n}\right) \in X^{n}$ such that $x^{1} \preceq y^{1}, y^{2} \prec x^{2}, x^{3} \preceq y^{3}, \ldots, y^{n} \prec x^{n}$. 
Theorem 3.1 Let $(X, \preceq)$ be a partially ordered set and suppose that there is a partial metric $p$ on $X$ such that $(X, p)$ is a complete partial metric space. Let $F: X^{n} \rightarrow X$ be a given mapping satisfying the following hypotheses:

(1) $F$ is continuous,

(2) F has the mixed strict monotone property,

(3) $F$ is a generalized Meir-Keeler type function,

(4) there exist $x_{0}^{1}, x_{0}^{2}, x_{0}^{3}, \ldots, x_{0}^{n} \in X$ such that

$$
\left\{\begin{array}{l}
x_{0}^{1} \prec F\left(x_{0}^{1}, x_{0}^{2}, x_{0}^{3}, \ldots, x_{0}^{n}\right), \\
F\left(x_{0}^{2}, x_{0}^{3}, \ldots, x_{0}^{n}, x_{0}^{1}\right) \preceq x_{0}^{2}, \\
x_{0}^{3} \prec F\left(x_{0}^{3}, \ldots, x_{0}^{n}, x_{0}^{1}, x_{0}^{2}\right), \\
\vdots \\
F\left(x_{0}^{n}, x_{0}^{1}, x_{0}^{2}, \ldots, x_{0}^{n-1}\right) \preceq x_{0}^{n} .
\end{array}\right.
$$

Then there exist $\left(x^{1}, x^{2}, x^{3}, \ldots, x^{n}\right) \in X^{n}$ such that $x^{1}=F\left(x^{1}, x^{2}, x^{3}, \ldots, x^{n}\right), x^{2}=F\left(x^{2}, x^{3}, \ldots\right.$, $\left.x^{n}, x^{1}\right), \ldots, x^{n}=F\left(x^{n}, x^{1}, x^{2}, \ldots, x^{n-1}\right)$.

Proof Let us define sequences $\left\{x_{m}^{1}\right\},\left\{x_{m}^{2}\right\}, \ldots,\left\{x_{m}^{n}\right\}$ in $X$ by

$$
\left\{\begin{array}{l}
x_{m}^{1}=F^{m}\left(x_{0}^{1}, x_{0}^{2}, x_{0}^{3}, \ldots, x_{0}^{n}\right), \\
x_{m}^{2}=F^{m}\left(x_{0}^{2}, x_{0}^{3}, \ldots, x_{0}^{n}, x_{0}^{1}\right), \\
x_{m}^{3}=F^{m}\left(x_{0}^{3}, \ldots, x_{0}^{n}, x_{0}^{1}, x_{0}^{2}\right), \\
\vdots \\
x_{m}^{n}=F^{m}\left(x_{0}^{n}, x_{0}^{1}, x_{0}^{2}, \ldots, x_{0}^{n-1}\right), \quad \forall m \in \mathbb{N} .
\end{array}\right.
$$

Since $F$ has mixed monotone property and from (3.3) we have

$$
\begin{aligned}
& x_{0}^{1} \prec x_{1}^{1} \prec x_{2}^{1} \prec \cdots \prec x_{m}^{1} \prec x_{m+1}^{1} \prec \cdots \\
& \cdots \preceq x_{m+1}^{2} \preceq x_{m}^{2} \preceq \cdots \preceq x_{2}^{2} \preceq x_{1}^{2} \preceq x_{0}^{2}, \\
& x_{0}^{3} \prec x_{1}^{3} \prec x_{2}^{3} \prec \cdots \prec x_{m}^{3} \prec x_{m+1}^{3} \prec \cdots \\
& \\
& \quad \vdots \\
& \cdots \\
& \quad \leq x_{m+1}^{n} \preceq x_{m}^{n} \preceq \cdots \preceq x_{2}^{n} \preceq x_{1}^{n} \preceq x_{0}^{n} .
\end{aligned}
$$

Applying Lemma 3.2 by taking $x^{1}=x_{0}^{1}, x^{2}=x_{0}^{2}, \ldots, x^{n}=x_{0}^{n}$ and $y^{1}=x_{1}^{1}, y^{2}=x_{1}^{2}, \ldots, y^{n}=x_{1}^{n}$, then we get

$$
\begin{aligned}
& \eta\left(\left(F^{m}\left(x_{0}^{1}, x_{0}^{2}, \ldots, x_{0}^{n}\right), F^{m}\left(x_{0}^{2}, \ldots, x_{0}^{n}, x_{0}^{1}\right), \ldots, F^{m}\left(x_{0}^{n}, x_{0}^{1}, \ldots, x_{0}^{n-1}\right)\right),\right. \\
& \left.\quad\left(F^{m}\left(x_{1}^{1}, x_{1}^{2}, \ldots, x_{1}^{n}\right), F^{m}\left(x_{1}^{2}, \ldots, x_{1}^{n}, x_{1}^{1}\right), \ldots, F^{m}\left(x_{1}^{n}, x_{1}^{1}, \ldots, x_{1}^{n-1}\right)\right)\right) \rightarrow 0 \quad \text { as } m \rightarrow \infty,
\end{aligned}
$$

that is,

$$
\eta\left(\left(x_{m}^{1}, x_{m}^{2}, \ldots, x_{m}^{n}\right),\left(x_{m+1}^{1}, x_{m+1}^{2}, \ldots, x_{m+1}^{n}\right)\right) \rightarrow 0 \quad \text { as } m \rightarrow \infty .
$$


Denote

$$
\begin{aligned}
& \eta^{s}\left(\left(x_{m}^{1}, x_{m}^{2}, \ldots, x_{m}^{n}\right),\left(x_{m+1}^{1}, x_{m+1}^{2}, \ldots, x_{m+1}^{n}\right)\right) \\
& \quad=2 \max \left\{p^{s}\left(x_{m}^{1}, x_{m+1}^{1}\right), p^{s}\left(x_{m}^{2}, x_{m+1}^{2}\right), \ldots, p^{s}\left(x_{m}^{n}, x_{m+1}^{n}\right)\right\}, \quad \forall m \in \mathbb{N} .
\end{aligned}
$$

From the definition of $p^{s}$, it is clear that

$$
\begin{aligned}
& \eta^{s}\left(\left(x_{m}^{1}, x_{m}^{2}, \ldots, x_{m}^{n}\right),\left(x_{m+1}^{1}, x_{m+1}^{2}, \ldots, x_{m+1}^{n}\right)\right) \\
& \quad \leq 2 \eta\left(\left(x_{m}^{1}, x_{m}^{2}, \ldots, x_{m}^{n}\right),\left(x_{m+1}^{1}, x_{m+1}^{2}, \ldots, x_{m+1}^{n}\right)\right), \quad \forall m \in \mathbb{N} .
\end{aligned}
$$

Using (3.11), we get

$$
\begin{aligned}
& \lim _{m \rightarrow \infty} \eta^{s}\left(\left(x_{m}^{1}, x_{m}^{2}, \ldots, x_{m}^{n}\right),\left(x_{m+1}^{1}, x_{m+1}^{2}, \ldots, x_{m+1}^{n}\right)\right) \\
& \quad=\lim _{m \rightarrow \infty} \max \left\{p^{s}\left(x_{m}^{1}, x_{m+1}^{1}\right), p^{s}\left(x_{m}^{2}, x_{m+1}^{2}\right), \ldots, p^{s}\left(x_{m}^{n}, x_{m+1}^{n}\right)\right\}=0 .
\end{aligned}
$$

Let $\epsilon>0$. It follows from (3.12) that there exists $k \in \mathbb{N}$ such that

$$
\eta^{s}\left(\left(x_{k}^{1}, x_{k}^{2}, \ldots, x_{k}^{n}\right),\left(x_{k+1}^{1}, x_{k+1}^{2}, \ldots, x_{k+1}^{n}\right)\right)<\delta(\epsilon) .
$$

Without restriction of generality, we can suppose that $\delta(\epsilon) \leq \epsilon$. We introduce the set $\wedge \subset$ $X^{n}$ defined by

$$
\begin{aligned}
\wedge:= & \left\{\left(x^{1}, x^{2}, x^{3}, \ldots, x^{n}\right) \in X^{n}: x_{k}^{1} \prec x^{1}, x^{2} \preceq x_{k}^{2}, x_{k}^{3} \prec x^{3}, \ldots, x^{n} \preceq x_{k}^{n},\right. \\
& \left.\eta^{s}\left(\left(x_{k}^{1}, x_{k}^{2}, x_{k}^{3}, \ldots, x_{k}^{n}\right),\left(x_{k+1}^{1}, x_{k+1}^{2}, x_{k+1}^{3}, \ldots, x_{k+1}^{n}\right)\right)<\epsilon+\delta(\epsilon)\right\} .
\end{aligned}
$$

Now we will prove that $\forall\left(x^{1}, x^{2}, x^{3}, \ldots, x^{n}\right) \in \wedge$,

$$
\left(F\left(x^{1}, x^{2}, x^{3}, \ldots, x^{n}\right), F\left(x^{2}, x^{3}, \ldots, x^{n}, x^{1}\right), \ldots, F\left(x^{n}, x^{1}, x^{2}, \ldots, x^{n-1}\right)\right) \in \wedge
$$

Let $\left(x^{1}, x^{2}, x^{3}, \ldots, x^{n}\right) \in \wedge$. We have

$$
\begin{aligned}
\eta^{s} & \left(\left(x_{k}^{1}, x_{k}^{2}, \ldots, x_{k}^{n}\right),\left(F\left(x^{1}, x^{2}, \ldots, x^{n}\right), F\left(x^{2}, \ldots, x^{n}, x^{1}\right), \ldots, F\left(x^{n}, x^{1}, \ldots, x^{n-1}\right)\right)\right) \\
= & \max \left\{p^{s}\left(x_{k}^{1}, F\left(x^{1}, x^{2}, \ldots, x^{n}\right)\right), p^{s}\left(x_{k}^{2}, F\left(x^{2}, \ldots, x^{n}, x^{1}\right)\right), \ldots, p^{s}\left(x_{k}^{n}, F\left(x^{n}, x^{1}, \ldots, x^{n-1}\right)\right)\right\} \\
\leq & \max \left\{p^{s}\left(x_{k}^{1}, x_{k+1}^{1}\right)+p^{s}\left(x_{k+1}^{1}, F\left(x^{1}, x^{2}, \ldots, x^{n}\right)\right), p^{s}\left(x_{k}^{2}, x_{k+1}^{2}\right)\right. \\
& \left.+p^{s}\left(x_{k+1}^{2}, F\left(x^{2}, \ldots, x^{n}, x^{1}\right)\right), \ldots, p^{s}\left(x_{k}^{n}, x_{k+1}^{n}\right)+p^{s}\left(x_{k+1}^{n}, F\left(x^{n}, x^{1}, \ldots, x^{n-1}\right)\right)\right\} \\
= & \max \left\{p^{s}\left(x_{k}^{1}, x_{k+1}^{1}\right)+p^{s}\left(F\left(x_{k}^{1}, x_{k}^{2}, \ldots, x_{k}^{n}\right), F\left(x^{1}, x^{2}, \ldots, x^{n}\right)\right), p^{s}\left(x_{k}^{2}, x_{k+1}^{2}\right)\right. \\
& +p^{s}\left(F\left(x_{k}^{2}, \ldots, x_{k}^{n}, x_{k}^{1}\right), F\left(x^{2}, \ldots, x^{n}, x^{1}\right)\right), \ldots, p^{s}\left(x_{k}^{n}, x_{k+1}^{n}\right) \\
& \left.+p^{s}\left(F\left(x_{k}^{n}, x_{k}^{1}, \ldots, x_{k}^{n-1}\right), F\left(x^{n}, x^{1}, \ldots, x^{n-1}\right)\right)\right\} \\
\leq & \max \left\{p^{s}\left(x_{k}^{1}, x_{k+1}^{1}\right), p^{s}\left(x_{k}^{2}, x_{k+1}^{2}\right), \ldots, p^{s}\left(x_{k}^{n}, x_{k+1}^{n}\right)\right\}+\max \left\{p ^ { s } \left(F\left(x_{k}^{1}, x_{k}^{2}, \ldots, x_{k}^{n}\right),\right.\right. \\
& \left.F\left(x^{1}, x^{2}, \ldots, x^{n}\right)\right), p^{s}\left(F\left(x_{k}^{2}, \ldots, x_{k}^{n}, x_{k}^{1}\right), F\left(x^{2}, \ldots, x^{n}, x^{1}\right)\right), \ldots, \\
& \left.p^{s}\left(F\left(x_{k}^{n}, x_{k}^{1}, \ldots, x_{k}^{n-1}\right), F\left(x^{n}, x^{1}, \ldots, x^{n-1}\right)\right)\right\}
\end{aligned}
$$




$$
\begin{aligned}
& <\delta(\epsilon)+\max \left\{p^{s}\left(F\left(x_{k}^{1}, x_{k}^{2}, \ldots, x_{k}^{n}\right), F\left(x^{1}, x^{2}, \ldots, x^{n}\right)\right), p^{s}\left(F\left(x_{k}^{2}, \ldots, x_{k}^{n}, x_{k}^{1}\right),\right.\right. \\
& \left.\left.\quad F\left(x^{2}, \ldots, x^{n}, x^{1}\right)\right), \ldots, p^{s}\left(F\left(x_{k}^{n}, x_{k}^{1}, \ldots, x_{k}^{n-1}\right), F\left(x^{n}, x^{1}, \ldots, x^{n-1}\right)\right)\right\} \quad \text { (by (3.13)). }
\end{aligned}
$$

We consider the following two cases.

Case I: $\eta^{s}\left(\left(x_{k}^{1}, x_{k}^{2}, \ldots, x_{k}^{n}\right),\left(x^{1}, x^{2}, \ldots, x^{n}\right)\right) \leq \epsilon$

By Lemma 3.1, we have

$$
\begin{aligned}
\eta^{s} & \left(\left(x_{k}^{1}, x_{k}^{2}, \ldots, x_{k}^{n}\right),\left(F\left(x^{1}, x^{2}, \ldots, x^{n}\right), F\left(x^{2}, \ldots, x^{n}, x^{1}\right), \ldots, F\left(x^{n}, x^{1}, \ldots, x^{n-1}\right)\right)\right) \\
< & \delta(\epsilon)+\max \left\{p^{s}\left(F\left(x_{k}^{1}, x_{k}^{2}, \ldots, x_{k}^{n}\right), F\left(x^{1}, x^{2}, \ldots, x^{n}\right)\right), p^{s}\left(F\left(x_{k}^{2}, \ldots, x_{k}^{n}, x_{k}^{1}\right),\right.\right. \\
& \left.\left.F\left(x^{2}, \ldots, x^{n}, x^{1}\right)\right), \ldots, p^{s}\left(F\left(x_{k}^{n}, x_{k}^{1}, \ldots, x_{k}^{n-1}\right), F\left(x^{n}, x^{1}, \ldots, x^{n-1}\right)\right)\right\} \\
< & \delta(\epsilon)+\max \left\{\max \left[p^{s}\left(x_{k}^{1}, x^{1}\right), p^{s}\left(x_{k}^{2}, x^{2}\right), \ldots, p^{s}\left(x_{k}^{n}, x^{n}\right)\right], \max \left[p^{s}\left(x_{k}^{2}, x^{2}\right), \ldots,\right.\right. \\
& \left.\left.p^{s}\left(x_{k}^{n}, x^{n}\right), p^{s}\left(x_{k}^{1}, x^{1}\right)\right], \ldots, \max \left[p^{s}\left(x_{k}^{n}, x^{n}\right), p^{s}\left(x_{k}^{1}, x^{1}\right), \ldots, p^{s}\left(x_{k}^{n-1}, x^{n-1}\right)\right]\right\} \\
< & \delta(\epsilon)+\eta^{s}\left(\left(x_{k}^{1}, x_{k}^{2}, \ldots, x_{k}^{n}\right),\left(x^{1}, x^{2}, \ldots, x^{n}\right)\right) \leq \delta(\epsilon)+\epsilon .
\end{aligned}
$$

Case II: $\epsilon+\eta^{s}\left(\left(x_{k}^{1}, x_{k}^{2}, \ldots, x_{k}^{n}\right),\left(x^{1}, x^{2}, \ldots, x^{n}\right)\right) \leq \delta(\epsilon)+\epsilon$.

We have

$$
\begin{gathered}
\eta^{s}\left(\left(x_{k}^{1}, x_{k}^{2}, \ldots, x_{k}^{n}\right),\left(F\left(x^{1}, x^{2}, \ldots, x^{n}\right), F\left(x^{2}, \ldots, x^{n}, x^{1}\right), \ldots, F\left(x^{n}, x^{1}, \ldots, x^{n-1}\right)\right)\right) \\
<\delta(\epsilon)+\max \left\{p^{s}\left(F\left(x_{k}^{1}, x_{k}^{2}, \ldots, x_{k}^{n}\right), F\left(x^{1}, x^{2}, \ldots, x^{n}\right)\right), p^{s}\left(F\left(x_{k}^{2}, \ldots, x_{k}^{n}, x_{k}^{1}\right),\right.\right. \\
\left.\left.F\left(x^{2}, \ldots, x^{n}, x^{1}\right)\right), \ldots, p^{s}\left(F\left(x_{k}^{n}, x_{k}^{1}, \ldots, x_{k}^{n-1}\right), F\left(x^{n}, x^{1}, \ldots, x^{n-1}\right)\right)\right\} .
\end{gathered}
$$

In this case, we get

$$
\epsilon<\max \left\{p^{s}\left(x_{k}^{1}, x^{1}\right), p^{s}\left(x_{k}^{2}, x^{2}\right), \ldots, p^{s}\left(x_{k}^{n}, x^{n}\right)\right\}<\epsilon+\delta(\epsilon) .
$$

Since $x_{k}^{1} \prec x^{1}, x^{2} \preceq x_{k}^{2}, x_{k}^{3} \prec x^{3}, \ldots, x^{n} \preceq x_{k}^{n}$, by (3) we get

$$
p^{s}\left(F\left(x^{1}, x^{2}, \ldots, x^{n}\right), F\left(x_{k}^{1}, x_{k}^{2}, \ldots, x_{k}^{n}\right)\right)<\epsilon .
$$

Also we have

$$
\epsilon<\max \left\{p^{s}\left(x_{k}^{2}, x^{2}\right), \ldots, p^{s}\left(x_{k}^{n}, x^{n}\right), p^{s}\left(x_{k}^{1}, x^{1}\right)\right\}<\epsilon+\delta(\epsilon) .
$$

By (3), this implies that

$$
p^{s}\left(F\left(x_{k}^{2}, \ldots, x_{k}^{n}, x_{k}^{1}\right), F\left(x^{2}, \ldots, x^{n}, x^{1}\right)\right)<\epsilon .
$$

In the same way we have

$$
p^{s}\left(F\left(x^{n}, x^{1}, \ldots, x^{n-1}\right), F\left(x_{k}^{n}, x_{k}^{1}, \ldots, x_{k}^{n-1}\right)\right)<\epsilon .
$$

Hence combining (3.15)-(3.18), we obtain

$$
\eta^{s}\left(\left(x_{k}^{1}, x_{k}^{2}, \ldots, x_{k}^{n}\right),\left(F\left(x_{k}^{1}, x_{k}^{2}, \ldots, x_{k}^{n}\right), F\left(x_{k}^{2}, \ldots, x_{k}^{n}, x_{k}^{1}\right), \ldots, F\left(x_{k}^{n}, x_{k}^{1}, \ldots, x_{k}^{n-1}\right)\right)\right)<\epsilon+\delta(\epsilon) .
$$


On the other hand, using (2), we can check easily that

$$
\begin{aligned}
& x_{k}^{1} \prec F\left(x^{1}, x^{2}, \ldots, x^{n}\right), \quad F\left(x^{2}, \ldots, x^{n}, x^{1}\right) \preceq x_{k}^{2}, \quad \ldots, \\
& F\left(x^{n}, x^{1}, \ldots, x^{n-1}\right) \preceq x_{k}^{n} .
\end{aligned}
$$

Hence, we deduce that (3.14) holds. By (3.13), we have $\left(x_{k+1}^{1}, x_{k+1}^{2}, \ldots, x_{k+1}^{n}\right) \in \wedge$. This implies with (3.14) that

$$
\begin{aligned}
& \left(x_{k+1}^{1}, x_{k+1}^{2}, \ldots, x_{k+1}^{n}\right) \in \wedge \\
& \Rightarrow \quad\left(F\left(x_{k+1}^{1}, x_{k+1}^{2}, \ldots, x_{k+1}^{n}\right), F\left(x_{k+1}^{2}, \ldots, x_{k+1}^{n}, x_{k+1}^{1}\right), \ldots, F\left(x_{k+1}^{n}, x_{k+1}^{1}, \ldots, x_{k+1}^{n-1}\right)\right) \\
& \quad=\left(x_{k+2}^{1}, x_{k+2}^{2}, \ldots, x_{k+2}^{n}\right) \in \wedge \\
& \Rightarrow \quad\left(F\left(x_{k+2}^{1}, x_{k+2}^{2}, \ldots, x_{k+2}^{n}\right), F\left(x_{k+2}^{2}, \ldots, x_{k+2}^{n}, x_{k+2}^{1}\right), \ldots, F\left(x_{k+2}^{n}, x_{k+2}^{1}, \ldots, x_{k+2}^{n-1}\right)\right) \\
& \quad=\left(x_{k+3}^{1}, x_{k+3}^{2}, \ldots, x_{k+3}^{n}\right) \in \wedge \\
& \quad \vdots \\
& \Rightarrow \quad\left(x_{m}^{1}, x_{m}^{2}, \ldots, x_{m}^{n}\right) \in \wedge .
\end{aligned}
$$

Thus for all $m>k$, we have $\left(x_{m}^{1}, x_{m}^{2}, \ldots, x_{m}^{n}\right) \in \wedge$. This implies that for all $m, l>k$, we have

$$
\begin{aligned}
\eta^{s} & \left(\left(x_{m}^{1}, x_{m}^{2}, \ldots, x_{m}^{n}\right),\left(x_{l}^{1}, x_{l}^{2}, \ldots, x_{l}^{n}\right)\right) \\
= & \max \left\{p^{s}\left(x_{m}^{1}, x_{l}^{1}\right), p^{s}\left(x_{m}^{2}, x_{l}^{2}\right), \ldots, p^{s}\left(x_{m}^{n}, x_{l}^{n}\right)\right\} \\
\leq & \max \left\{p^{s}\left(x_{m}^{1}, x_{k}^{1}\right)+p^{s}\left(x_{k}^{1}, x_{l}^{1}\right), p^{s}\left(x_{m}^{2}, x_{k}^{2}\right)+p^{s}\left(x_{k}^{2}, x_{l}^{2}\right), \ldots, p^{s}\left(x_{m}^{n}, x_{k}^{n}\right)+p^{s}\left(x_{k}^{n}, x_{l}^{n}\right)\right\} \\
\leq & \max \left\{p^{s}\left(x_{m}^{1}, x_{k}^{1}\right), p^{s}\left(x_{m}^{2}, x_{k}^{2}\right), \ldots, p^{s}\left(x_{m}^{n}, x_{k}^{n}\right)\right\} \\
& \quad+\max \left\{p^{s}\left(x_{k}^{1}, x_{l}^{1}\right), p^{s}\left(x_{k}^{2}, x_{l}^{2}\right), \ldots, p^{s}\left(x_{k}^{n}, x_{l}^{n}\right)\right\} \\
= & \eta\left(\left(x_{m}^{1}, x_{m}^{2}, \ldots, x_{m}^{n}\right),\left(x_{k}^{1}, x_{k}^{2}, \ldots, x_{k}^{n}\right)\right)+\eta\left(\left(x_{k}^{1}, x_{k}^{2}, \ldots, x_{k}^{n}\right),\left(x_{m}^{1}, x_{m}^{2}, \ldots, x_{m}^{n}\right)\right) \\
< & 2(\epsilon+\delta(\epsilon))<4 \epsilon .
\end{aligned}
$$

We deduce that $\left\{\left(x_{m}^{1}, x_{m}^{2}, \ldots, x_{m}^{n}\right)\right\}$ is a Cauchy sequence in the metric space $\left(X^{n}, \eta^{s}\right)$. Since $(X, p)$ is complete, from Lemma $2.1,\left(X, p^{s}\right)$ is a complete metric space. Therefore $\left(X^{n}, \eta^{s}\right)$ is complete. Hence there exist $\left(x^{1}, x^{2}, \ldots, x^{n}\right) \in X^{n}$ such that

$$
\eta^{s}\left(\left(x_{m}^{1}, x_{m}^{2}, \ldots, x_{m}^{n}\right),\left(x^{1}, x^{2}, \ldots, x^{n}\right)\right) \rightarrow 0 \quad \text { as } m \rightarrow \infty
$$

which shows that

$$
\begin{aligned}
& p^{s}\left(F^{m}\left(x_{0}^{1}, x_{0}^{2}, \ldots, x_{0}^{n}\right), x^{1}\right) \rightarrow 0 \quad \text { as } m \rightarrow \infty, \\
& p^{s}\left(F^{m}\left(x_{0}^{2}, \ldots, x_{0}^{n}, x_{0}^{1}\right), x^{2}\right) \rightarrow 0 \quad \text { as } m \rightarrow \infty, \\
& \vdots \\
& p^{s}\left(F^{m}\left(x_{0}^{n}, x_{0}^{1}, \ldots, x_{0}^{n-1}\right), x^{n}\right) \rightarrow 0 \quad \text { as } m \rightarrow \infty .
\end{aligned}
$$


Therefore from Lemma 2.1 and using (3.12), we have

$$
\left\{\begin{array}{l}
p\left(x^{1}, x^{1}\right)=\lim _{m \rightarrow \infty} p\left(x_{m}^{1}, x^{1}\right)=\lim _{m \rightarrow \infty} p\left(x_{m}^{1}, x_{m}^{1}\right)=0, \\
p\left(x^{2}, x^{2}\right)=\lim _{m \rightarrow \infty} p\left(x_{m}^{2}, x^{2}\right)=\lim _{m \rightarrow \infty} p\left(x_{m}^{2}, x_{m}^{2}\right)=0, \\
\vdots \\
p\left(x^{n}, x^{n}\right)=\lim _{m \rightarrow \infty} p\left(x_{m}^{n}, x^{n}\right)=\lim _{m \rightarrow \infty} p\left(x_{m}^{n}, x_{m}^{n}\right)=0 .
\end{array}\right.
$$

We will show that $x^{1}=F\left(x^{1}, x^{2}, \ldots, x^{n}\right), x^{2}=F\left(x^{2}, \ldots, x^{n}, x^{1}\right), \ldots, x^{n}=F\left(x^{n}, x^{1}, \ldots, x^{n-1}\right)$. Since $F$ is continuous on $X$, then $F$ is continuous at $\left(x^{1}, x^{2}, \ldots, x^{n}\right)$. Hence for any $\epsilon>0$, there exists $\delta(\epsilon)>0$ such that if $\left(y^{1}, y^{2}, \ldots, y^{n}\right) \in X^{n}$ verifying

$$
\eta\left(\left(x^{1}, x^{2}, \ldots, x^{n}\right),\left(y^{1}, y^{2}, \ldots, y^{n}\right)\right)<\eta\left(\left(x^{1}, x^{2}, \ldots, x^{n}\right),\left(x^{1}, x^{2}, \ldots, x^{n}\right)\right)+\delta(\epsilon)
$$

means that

$$
\begin{aligned}
& \max \left\{p\left(x^{1}, y^{1}\right), p\left(x^{2}, y^{2}\right), \ldots, p\left(x^{n}, y^{n}\right)\right\} \\
& \quad<\max \left\{p\left(x^{1}, x^{1}\right), p\left(x^{2}, x^{2}\right), \ldots, p\left(x^{n}, x^{n}\right)\right\}+\delta(\epsilon)=\delta(\epsilon)
\end{aligned}
$$

because $p\left(x^{1}, x^{1}\right)=p\left(x^{2}, x^{2}\right)=\cdots=p\left(x^{n}, x^{n}\right)=0$, then we have

$$
p\left(F\left(x^{1}, x^{2}, \ldots, x^{n}\right), F\left(y^{1}, y^{2}, \ldots, y^{n}\right)\right)<p\left(F\left(x^{1}, x^{2}, \ldots, x^{n}\right), F\left(x^{1}, x^{2}, \ldots, x^{n}\right)\right)+\frac{\epsilon}{2} .
$$

Since

$$
\lim _{m \rightarrow \infty} p\left(x_{m}^{1}, x^{1}\right)=\lim _{m \rightarrow \infty} p\left(x_{m}^{2}, x^{2}\right)=\cdots=\lim _{m \rightarrow \infty} p\left(x_{m}^{n}, x^{n}\right)=0
$$

for $\alpha=\min \left\{\frac{\delta(\epsilon)}{2}, \frac{\epsilon}{2}\right\}>0$, there exists $m_{0}, l_{0} \in \mathbb{N}$ such that for $m>m_{0}, l>l_{0}, p\left(x_{m}^{1}, x^{1}\right)<$ $\alpha, p\left(x_{m}^{2}, x^{2}\right)<\alpha, \ldots, p\left(x_{m}^{n}, x^{n}\right)<\alpha$. Then for $m \in \mathbb{N}, m \geq \max \left\{m_{0}, l_{0}\right\}$, we have

$$
\max \left\{p\left(x_{m}^{1}, x^{1}\right), p\left(x_{m}^{2}, x^{2}\right), \ldots, p\left(x_{m}^{n}, x^{n}\right)\right\}<\alpha<\frac{\delta(\epsilon)}{2}
$$

so we get

$$
p\left(F\left(x^{1}, x^{2}, \ldots, x^{n}\right), F\left(x_{m}^{1}, x_{m}^{2}, \ldots, x_{m}^{n}\right)\right)<p\left(F\left(x^{1}, x^{2}, \ldots, x^{n}\right), F\left(x^{1}, x^{2}, \ldots, x^{n}\right)\right)+\frac{\epsilon}{2} .
$$

Now, for any $m \geq \max \left\{m_{0}, l_{0}\right\}$,

$$
\begin{aligned}
p\left(F\left(x^{1}, x^{2}, \ldots, x^{n}\right), x^{1}\right) & \leq p\left(F\left(x^{1}, x^{2}, \ldots, x^{n}\right), x_{m+1}^{1}\right)+p\left(x_{m+1}^{1}, x^{1}\right) \\
& =p\left(F\left(x^{1}, x^{2}, \ldots, x^{n}\right), F\left(x_{m}^{1}, x_{m}^{2}, \ldots, x_{m}^{n}\right)\right)+p\left(x_{m+1}^{1}, x^{1}\right) \\
& <p\left(F\left(x^{1}, x^{2}, \ldots, x^{n}\right), F\left(x^{1}, x^{2}, \ldots, x^{n}\right)\right)+\frac{\epsilon}{2}+\alpha \quad \text { (by (3.20)) } \\
& <p\left(F\left(x^{1}, x^{2}, \ldots, x^{n}\right), F\left(x^{1}, x^{2}, \ldots, x^{n}\right)\right)+\epsilon .
\end{aligned}
$$


On the other hand, since $F$ is a generalized Meir-Keeler type function, then from Lemma 3.1, we have

$$
p\left(F\left(x^{1}, x^{2}, \ldots, x^{n}\right), F\left(x^{1}, x^{2}, \ldots, x^{n}\right)\right)<\max \left\{p\left(x^{1}, x^{1}\right), p\left(x^{2}, x^{2}\right), \ldots, p\left(x^{n}, x^{n}\right)\right\}=0 .
$$

In this case, for any $\epsilon>0, p\left(F\left(x^{1}, x^{2}, \ldots, x^{n}\right), x^{1}\right)<\epsilon$. This implies that $F\left(x^{1}, x^{2}, \ldots, x^{n}\right)=x^{1}$. Similarly we can show that

$$
F\left(x^{2}, \ldots, x^{n}, x^{1}\right)=x^{2}, \quad \ldots, \quad F\left(x^{n}, x^{1}, \ldots, x^{n-1}\right)=x^{n} .
$$

Thus we have proved that $F$ has an $n$-tupled fixed point.

Remark 3.2 Theorem 3.1 still holds if we replace (3.10) by $\exists x_{0}^{1}, x_{0}^{2}, x_{0}^{3}, \ldots, x_{0}^{n} \in X$ such that

$$
\left\{\begin{array}{l}
x_{0}^{1} \preceq F\left(x_{0}^{1}, x_{0}^{2}, x_{0}^{3}, \ldots, x_{0}^{n}\right), \\
F\left(x_{0}^{2}, x_{0}^{3}, \ldots, x_{0}^{n}, x_{0}^{1}\right) \prec x_{0}^{2}, \\
x_{0}^{3} \preceq F\left(x_{0}^{3}, \ldots, x_{0}^{n}, x_{0}^{1}, x_{0}^{2}\right), \\
\vdots \\
F\left(x_{0}^{n}, x_{0}^{1}, x_{0}^{2}, \ldots, x_{0}^{n-1}\right) \prec x_{0}^{n} .
\end{array}\right.
$$

Theorem 3.2 Let $(X, \preceq)$ be a partially ordered set and suppose that there is a partial metric $p$ on $X$ such that $(X, p)$ is a complete partial metric space. Assume that $X$ has the following properties:

(a) if a nondecreasing sequence $x_{m} \rightarrow x$ then $x_{m} \preceq x$ for all $m \geq 0$,

(b) if a nonincreasing sequence $x_{m} \rightarrow x$ then $x \leq x_{m}$ for all $m \geq 0$.

Let $F: X^{n} \rightarrow X$ be a given mapping satisfying the following hypotheses:

(1) $F$ is continuous,

(2) F has the mixed strict monotone property,

(3) $F$ is a generalized Meir-Keeler type function,

(4) there exist $x_{0}^{1}, x_{0}^{2}, x_{0}^{3}, \ldots, x_{0}^{n} \in X$ such that (3.10) holds.

Then there exists $\left(x^{1}, x^{2}, x^{3}, \ldots, x^{n}\right) \in X^{n}$ such that $x^{1}=F\left(x^{1}, x^{2}, x^{3}, \ldots, x^{n}\right), x^{2}=F\left(x^{2}\right.$, $\left.x^{3}, \ldots, x^{n}, x^{1}\right), \ldots, x^{n}=F\left(x^{n}, x^{1}, x^{2}, \ldots, x^{n-1}\right)$. Furthermore, $p\left(x^{1}, x^{1}\right)=p\left(x^{2}, x^{2}\right)=\cdots=p\left(x^{n}\right.$, $\left.x^{n}\right)=0$.

Proof Following the proof of Theorem 3.1, we only have to prove that

$$
\begin{aligned}
& x^{1}=F\left(x^{1}, x^{2}, x^{3}, \ldots, x^{n}\right), \quad x^{2}=F\left(x^{2}, x^{3}, \ldots, x^{n}, x^{1}\right), \\
& x^{n}=F\left(x^{n}, x^{1}, x^{2}, \ldots, x^{n-1}\right) .
\end{aligned}
$$

Let $\epsilon>0$. Since $F^{m}\left(x_{0}^{1}, x_{0}^{2}, \ldots, x_{0}^{n}\right) \rightarrow x^{1}, F^{m}\left(x_{0}^{2}, \ldots, x_{0}^{n}, x_{0}^{1}\right) \rightarrow x^{2}, \ldots, F^{m}\left(x_{0}^{n}, x_{0}^{1}, \ldots, x_{0}^{n-1}\right) \rightarrow$ $x^{n}$. Then there exist $m_{1}, m_{2}, \ldots, m_{n} \in \mathbb{N}^{*}$ such that for all $m \geq m_{1}, l \geq m_{2}, \ldots, t \geq m_{n}$,

$$
\left\{\begin{array}{l}
p\left(F^{m}\left(x_{0}^{1}, x_{0}^{2}, \ldots, x_{0}^{n}\right), x^{1}\right)<\epsilon, \\
p\left(F^{l}\left(x_{0}^{2}, \ldots, x_{0}^{n}, x_{0}^{1}\right), x^{2}\right)<\epsilon, \\
\vdots \\
p\left(F^{t}\left(x_{0}^{n}, x_{0}^{1}, \ldots, x_{0}^{n-1}\right), x^{n}\right)<\epsilon .
\end{array}\right.
$$


Taking $m \geq \max \left\{m_{1}, m_{2}, \ldots, m_{n}\right\}$ and using

$$
\begin{aligned}
& F^{m}\left(x_{0}^{1}, x_{0}^{2}, \ldots, x_{0}^{n}\right) \prec x^{1}, \quad x^{2} \prec F^{m}\left(x_{0}^{2}, \ldots, x_{0}^{n}, x_{0}^{1}\right), \\
& x^{n} \prec F^{m}\left(x_{0}^{n}, x_{0}^{1}, \ldots, x_{0}^{n-1}\right),
\end{aligned}
$$

by (3.21) and Lemma 3.1, we get

$$
\begin{aligned}
p\left(F\left(x^{1}, x^{2}, \ldots, x^{n}\right), x^{1}\right) \leq & p\left(F\left(x^{1}, x^{2}, \ldots, x^{n}\right), F^{m+1}\left(x_{0}^{1}, x_{0}^{2}, \ldots, x_{0}^{n}\right)\right) \\
& +p\left(F^{m+1}\left(x_{0}^{1}, x_{0}^{2}, \ldots, x_{0}^{n}\right), x^{1}\right) \\
= & p\left(F\left(x^{1}, x^{2}, \ldots, x^{n}\right), F\left(F^{m}\left(x_{0}^{1}, x_{0}^{2}, \ldots, x_{0}^{n}\right),\right.\right. \\
& F^{m}\left(x_{0}^{2}, \ldots, x_{0}^{n}, x_{0}^{1}\right), \ldots, \\
& \left.\left.F^{m}\left(x_{0}^{n}, x_{0}^{1}, \ldots, x_{0}^{n-1}\right)\right)\right)+p\left(F^{m+1}\left(x_{0}^{1}, x_{0}^{2}, \ldots, x_{0}^{n}\right), x^{1}\right) \\
< & \max \left\{p\left(x^{1}, F^{m}\left(x_{0}^{1}, x_{0}^{2}, \ldots, x_{0}^{n}\right)\right), p\left(x^{2}, F^{m}\left(x_{0}^{2}, \ldots, x_{0}^{n}, x_{0}^{1}\right)\right), \ldots,\right. \\
& \left.p\left(x^{n}, F^{m}\left(x_{0}^{n}, x_{0}^{1}, \ldots, x_{0}^{n-1}\right)\right)\right\}+p\left(F^{m+1}\left(x_{0}^{1}, x_{0}^{2}, \ldots, x_{0}^{n}\right), x^{1}\right) \\
< & 2 \epsilon .
\end{aligned}
$$

This implies that $F\left(x^{1}, x^{2}, \ldots, x^{n}\right)=x^{1}$. Similarly, we can show that

$$
p\left(F\left(x^{2}, \ldots, x^{n}, x^{1}\right), x^{2}\right)<2 \epsilon, \quad \ldots, \quad p\left(F\left(x^{n}, x^{1}, \ldots, x^{n-1}\right), x^{n}\right)<2 \epsilon
$$

which implies that $F\left(x^{2}, \ldots, x^{n}, x^{1}\right)=x^{2}, \ldots, F\left(x^{n}, x^{1}, \ldots, x^{n-1}\right)=x^{n}$.

This completes the proof.

Now we endow the product space $X^{n}$ with the following partial order: for $\left(x^{1}, x^{2}, \ldots, x^{n}\right)$, $\left(y^{1}, y^{2}, \ldots, y^{n}\right) \in X^{n}$,

$$
\begin{aligned}
& \left(y^{1}, y^{2}, \ldots, y^{n}\right) \preceq\left(x^{1}, x^{2}, \ldots, x^{n}\right) \\
& \quad \Leftrightarrow \quad y^{1} \preceq x^{1}, \quad x^{2} \preceq y^{2}, \quad y^{3} \preceq x^{3}, \quad \ldots, \quad x^{n} \preceq y^{n} .
\end{aligned}
$$

One can prove that $n$-tupled fixed point is in fact unique and the product space $X^{n}$ endow with this partial order has the following property:

(A) $\forall\left(x^{1}, x^{2}, \ldots, x^{n}\right),\left(z^{1}, z^{2}, \ldots, z^{n}\right) \in X^{n}, \exists\left(t^{1}, t^{2}, \ldots, t^{n}\right) \in X^{n}$ that is comparable to $\left(x^{1}, x^{2}, \ldots, x^{n}\right)$ and $\left(z^{1}, z^{2}, \ldots, z^{n}\right)$.

Theorem 3.3 Adding (A) to the hypotheses of Theorem 3.1 (respectively, Theorem 3.2), we obtain the uniqueness of n-tupled fixed point of $F$.

Proof Suppose that $\left(z^{1}, z^{2}, \ldots, z^{n}\right) \in X^{n}$ is another $n$-tupled fixed point of $F$. We distinguish two cases:

Case I: $\left(x^{1}, x^{2}, \ldots, x^{n}\right)$ is comparable to $\left(z^{1}, z^{2}, \ldots, z^{n}\right)$ with respect to ordering in $X^{n}$, where

$$
\begin{aligned}
& \lim _{m \rightarrow \infty} F^{m}\left(x_{0}^{1}, x_{0}^{2}, \ldots, x_{0}^{n}\right)=x^{1}, \quad \lim _{m \rightarrow \infty} F^{m}\left(x_{0}^{2}, \ldots, x_{0}^{n}, x_{0}^{1}\right)=x^{2}, \quad \ldots, \\
& \lim _{m \rightarrow \infty} F^{m}\left(x_{0}^{n}, x_{0}^{1}, \ldots, x_{0}^{n-1}\right)=x^{n} .
\end{aligned}
$$


Without restriction of generality, we can suppose that

$$
\begin{aligned}
& F\left(z^{1}, z^{2}, \ldots, z^{n}\right)=z^{1} \prec x^{1}=F\left(x^{1}, x^{2}, \ldots, x^{n}\right), \\
& F\left(x^{2}, \ldots, x^{n}, x^{1}\right)=x^{2} \preceq z^{2}=F\left(z^{2}, \ldots, z^{n}, z^{1}\right), \\
& \vdots \\
& F\left(x^{n}, x^{1}, \ldots, x^{n-1}\right)=x^{n} \preceq z^{n}=F\left(z^{n}, z^{1}, \ldots, z^{n-1}\right) .
\end{aligned}
$$

We have

$$
\begin{aligned}
\eta( & \left.\left(x^{1}, x^{2}, \ldots, x^{n}\right),\left(z^{1}, z^{2}, \ldots, z^{n}\right)\right) \\
= & \max \left\{p\left(x^{1}, z^{1}\right), p\left(x^{2}, z^{2}\right), \ldots, p\left(x^{n}, z^{n}\right)\right\} \\
= & \max \left\{p\left(F\left(x^{1}, x^{2}, \ldots, x^{n}\right), F\left(z^{1}, z^{2}, \ldots, z^{n}\right)\right), p\left(F\left(x^{2}, \ldots, x^{n}, x^{1}\right),\right.\right. \\
& \left.\left.F\left(z^{2}, \ldots, z^{n}, z^{1}\right)\right), \ldots, p\left(F\left(x^{n}, x^{1}, \ldots, x^{n-1}\right), F\left(z^{n}, z^{1}, \ldots, z^{n-1}\right)\right)\right\} \\
< & \max \left\{\max \left[p\left(x^{1}, z^{1}\right), p\left(x^{2}, z^{2}\right), \ldots, p\left(x^{n}, z^{n}\right)\right], \max \left[p\left(x^{1}, z^{1}\right), p\left(x^{2}, z^{2}\right), \ldots,\right.\right. \\
& \left.\left.p\left(x^{n}, z^{n}\right)\right], \ldots, \max \left[p\left(x^{1}, z^{1}\right), p\left(x^{2}, z^{2}\right), \ldots, p\left(x^{n}, z^{n}\right)\right]\right\} \\
= & \eta\left(\left(x^{1}, x^{2}, \ldots, x^{n}\right),\left(z^{1}, z^{2}, \ldots, z^{n}\right)\right) .
\end{aligned}
$$

Case II: $\left(x^{1}, x^{2}, \ldots, x^{n}\right)$ is not comparable to $\left(z^{1}, z^{2}, \ldots, z^{n}\right)$. Then there exists $\left(t^{1}, t^{2}, \ldots\right.$, $\left.t^{n}\right) \in X^{n}$ that is comparable to $\left(x^{1}, x^{2}, \ldots, x^{n}\right)$ and $\left(z^{1}, z^{2}, \ldots, z^{n}\right)$. Without restriction of generality, we can assume that

$$
\begin{aligned}
& x^{1} \prec t^{1}, \quad t^{2} \preceq x^{2}, \quad x^{3} \prec t^{3}, \quad \ldots, \quad t^{n} \preceq x^{n} \quad \text { and } \\
& z^{1} \prec t^{1}, \quad t^{2} \preceq z^{2}, \quad z^{3} \prec t^{3}, \quad \ldots, \quad t^{n} \preceq z^{n} .
\end{aligned}
$$

From (3.22) and Lemma 3.2, we have

$$
\begin{aligned}
& \eta\left(\left(F^{m}\left(x^{1}, x^{2}, \ldots, x^{n}\right), F^{m}\left(x^{2}, \ldots, x^{n}, x^{1}\right), \ldots, F^{m}\left(x^{n}, x^{1}, \ldots, x^{n-1}\right)\right),\left(F^{m}\left(t^{1}, t^{2}, \ldots, t^{n}\right),\right.\right. \\
& \left.\left.F^{m}\left(t^{2}, \ldots, t^{n}, t^{1}\right), \ldots, F^{m}\left(t^{n}, t^{1}, \ldots, t^{n-1}\right)\right)\right) \rightarrow 0 \quad \text { as } m \rightarrow \infty .
\end{aligned}
$$

Similarly we have

$$
\begin{aligned}
& \eta\left(\left(F^{m}\left(z^{1}, z^{2}, \ldots, z^{n}\right), F^{m}\left(z^{2}, \ldots, z^{n}, z^{1}\right), \ldots, F^{m}\left(z^{n}, z^{1}, \ldots, z^{n-1}\right)\right),\left(F^{m}\left(t^{1}, t^{2}, \ldots, t^{n}\right),\right.\right. \\
& \left.\left.F^{m}\left(t^{2}, \ldots, t^{n}, t^{1}\right), \ldots, F^{m}\left(t^{n}, t^{1}, \ldots, t^{n-1}\right)\right)\right) \rightarrow 0 \quad \text { as } m \rightarrow \infty .
\end{aligned}
$$

On the other hand, using the triangular inequality, we get

$$
\begin{aligned}
\eta & \left(\left(x^{1}, x^{2}, \ldots, x^{n}\right),\left(z^{1}, z^{2}, \ldots, z^{n}\right)\right) \\
= & \eta\left(\left(F^{m}\left(x^{1}, x^{2}, \ldots, x^{n}\right), F^{m}\left(x^{2}, \ldots, x^{n}, x^{1}\right), \ldots, F^{m}\left(x^{n}, x^{1}, \ldots, x^{n-1}\right)\right),\right. \\
& \left.\left(F^{m}\left(z^{1}, z^{2}, \ldots, z^{n}\right), F^{m}\left(z^{2}, \ldots, z^{n}, z^{1}\right), \ldots, F^{m}\left(z^{n}, z^{1}, \ldots, z^{n-1}\right)\right)\right) \\
\leq & \eta\left(\left(F^{m}\left(x^{1}, x^{2}, \ldots, x^{n}\right), F^{m}\left(x^{2}, \ldots, x^{n}, x^{1}\right), \ldots, F^{m}\left(x^{n}, x^{1}, \ldots, x^{n-1}\right)\right),\right.
\end{aligned}
$$




$$
\begin{aligned}
& \left.\left(F^{m}\left(t^{1}, t^{2}, \ldots, t^{n}\right), F^{m}\left(t^{2}, \ldots, t^{n}, t^{1}\right), \ldots, F^{m}\left(t^{n}, t^{1}, \ldots, t^{n-1}\right)\right)\right) \\
& +\eta\left(\left(F^{m}\left(t^{1}, t^{2}, \ldots, t^{n}\right), F^{m}\left(t^{2}, \ldots, t^{n}, t^{1}\right), \ldots, F^{m}\left(t^{n}, t^{1}, \ldots, t^{n-1}\right)\right),\right. \\
& \left.\left(F^{m}\left(z^{1}, z^{2}, \ldots, z^{n}\right), F^{m}\left(z^{2}, \ldots, z^{n}, z^{1}\right), \ldots, F^{m}\left(z^{n}, z^{1}, \ldots, z^{n-1}\right)\right)\right) .
\end{aligned}
$$

By (3.22) and (3.23), we have $\eta\left(\left(x^{1}, x^{2}, \ldots, x^{n}\right),\left(z^{1}, z^{2}, \ldots, z^{n}\right)\right)=0$, we get

$$
\left(x^{1}, x^{2}, \ldots, x^{n}\right)=\left(z^{1}, z^{2}, \ldots, z^{n}\right) .
$$

This completes the proof.

\section{Applications}

In this section, using the earlier results proved in the preceding section, we obtain some $n$-tupled fixed point theorem for mappings satisfying a general contractive condition of integral type in partially ordered complete partial metric spaces.

Theorem 4.1 Let $(X, \preceq)$ be a partially ordered set and suppose that there is a partial metric $p$ on $X$ such that $(X, p)$ is a complete partial metric space. Let $F: X^{n} \rightarrow X$ be a given mapping. Assume that there exists a function $\theta$ from $[0, \infty)$ into itself satisfying the following:

(1) $\theta(0)=0$ and $\theta(t)>0$ for every $t>0$,

(2) $\theta$ is nondecreasing and right continuous,

(3) for every $\epsilon>0$, there exists $\delta(\epsilon)>0$ such that

$$
\begin{gathered}
\epsilon<\theta\left(\max \left\{p\left(x^{1}, y^{1}\right), p\left(x^{2}, y^{2}\right), \ldots, p\left(x^{n}, y^{n}\right)\right\}\right)<\epsilon+\delta(\epsilon) \\
\Rightarrow \quad \theta\left(p\left(F\left(x^{1}, x^{2}, \ldots, x^{n}\right), F\left(y^{1}, y^{2}, \ldots, y^{n}\right)\right)\right)<\epsilon
\end{gathered}
$$

for all $y^{1} \prec x^{1}, x^{2} \preceq y^{2}, y^{3} \prec x^{3}, \ldots, x^{n} \preceq y^{n}$.

Then $F$ is a generalized Meir-Keeler type function.

Proof Fix $\epsilon>0$. Since $\theta(\epsilon)>0$, there exists $\alpha>0$ and $\left(a^{1}, a^{2}, \ldots, a^{n}\right),\left(b^{1}, b^{2}, \ldots, b^{n}\right) \in X^{n}$ such that

$$
\begin{aligned}
& \theta(\epsilon) \leq \theta\left(\max \left\{p\left(a^{1}, b^{1}\right), p\left(a^{2}, b^{2}\right), \ldots, p\left(a^{n}, b^{n}\right)\right\}\right)<\theta(\epsilon)+\delta(\epsilon) \\
& \quad \Rightarrow \quad \theta\left(p\left(F\left(a^{1}, a^{2}, \ldots, a^{n}\right), F\left(b^{1}, b^{2}, \ldots, b^{n}\right)\right)\right)<\epsilon .
\end{aligned}
$$

From the right continuity of $\theta$, there exists $\delta>0$ such that $\theta(\epsilon+\delta)<\theta(\epsilon)+\alpha$. Fix $x^{1}, x^{2}, \ldots, x^{n}, y^{1}, y^{2}, \ldots, y^{n} \in X$, then

$$
\epsilon \leq \max \left\{p\left(x^{1}, y^{1}\right), p\left(x^{2}, y^{2}\right), \ldots, p\left(x^{n}, y^{n}\right)\right\}<\epsilon+\delta .
$$

Since $\theta$ is a nondecreasing function, we get

$$
\theta(\epsilon) \leq \theta\left(\max \left\{p\left(x^{1}, y^{1}\right), p\left(x^{2}, y^{2}\right), \ldots, p\left(x^{n}, y^{n}\right)\right\}\right)<\theta(\epsilon+\delta)<\theta(\epsilon)+\alpha .
$$

By (4.1) we get

$$
\theta\left(p\left(F\left(x^{1}, x^{2}, \ldots, x^{n}\right), F\left(y^{1}, y^{2}, \ldots, y^{n}\right)\right)\right)<\theta(\epsilon),
$$


and hence

$$
p\left(F\left(x^{1}, x^{2}, \ldots, x^{n}\right), F\left(y^{1}, y^{2}, \ldots, y^{n}\right)\right)<\epsilon .
$$

The following result is an immediate consequence of Theorems 3.1, 3.2 and 4.1.

Corollary 4.1 Let $(X, \preceq)$ be a partially ordered set and suppose that there is a partial metric $p$ on $X$ such that $(X, p)$ is a complete partial metric space. Let $F: X^{n} \rightarrow X$ be a given mapping satisfying the following hypotheses:

(1) $F$ is continuous,

(2) $F$ has the mixed strict monotone property,

(3) for all $\epsilon>0$, there exists $\delta(\epsilon)>0$ such that

$$
\begin{aligned}
\epsilon & \leq \int_{0}^{\max \left\{p\left(x^{1}, y^{1}\right), p\left(x^{2}, y^{2}\right) \ldots, \ldots\left(x^{n}, y^{n}\right)\right\}} \varphi(t) d t<\epsilon+\delta(\epsilon) \\
& \Rightarrow \quad \int_{0}^{p\left(F\left(x^{1}, \ldots, x^{n}\right), F\left(y^{1}, \ldots, y^{n}\right)\right)} \varphi(t) d t<\epsilon
\end{aligned}
$$

for all $y^{1} \prec x^{1}, x^{2} \preceq y^{2}, y^{3} \prec x^{3}, \ldots, x^{n} \preceq y^{n}$, where $\varphi$ is a locally integrable function from $[0, \infty)$ into itself satisfying

$$
\int_{0}^{s} \varphi(t) d t>0, \quad \forall s>0,
$$

(4) $\exists x_{0}^{1}, x_{0}^{2}, x_{0}^{3}, \ldots, x_{0}^{n} \in X$ such that (3.10) holds.

Then there exists $\left(x^{1}, x^{2}, \ldots, x^{n}\right) \in X^{n}$ such that

$$
x^{1}=F\left(x^{1}, x^{2}, \ldots, x^{n}\right), \quad x^{2}=F\left(x^{2}, \ldots, x^{n}, x^{1}\right), \quad \ldots, \quad x^{n}=F\left(x^{n}, x^{1}, \ldots, x^{n-1}\right) .
$$

Moreover, if property (A) is satisfied, then the n-tupled fixed point of $F$ remains unique.

Remark 4.1 The conclusions of the preceding corollary remain valid if we replace the continuity hypothesis of $F$ by hypotheses (a) and (b) of Theorem 3.2.

Corollary 4.2 Let $(X, \preceq)$ be a partially ordered set and suppose that there is a partial metric $p$ on $X$ such that $(X, p)$ is a complete partial metric space. Let $F: X^{n} \rightarrow X$ be a given mapping satisfying the following hypotheses:

(1) $F$ is continuous,

(2) F has the mixed strict monotone property,

(3) for all $y^{1} \prec x^{1}, x^{2} \preceq y^{2}, y^{3} \prec x^{3}, \ldots, x^{n} \preceq y^{n}$,

$$
\int_{0}^{p\left(F\left(x^{1}, x^{2}, \ldots, x^{n}\right), F\left(y^{1}, y^{2}, \ldots, y^{n}\right)\right)} \varphi(t) d t \leq k \int_{0}^{\max \left\{p\left(x^{1}, y^{1}\right), p\left(x^{2}, y^{2}\right), \ldots, p\left(x^{n}, y^{n}\right)\right\}} \varphi(t) d t,
$$

where $k \in(0,1)$ and $\varphi$ is a locally integrable function from $[0, \infty)$ into itself satisfying

$$
\int_{0}^{s} \varphi(t) d t>0, \quad \forall s>0
$$

(4) $\exists x_{0}^{1}, x_{0}^{2}, x_{0}^{3}, \ldots, x_{0}^{n} \in X$ such that (3.10) holds. 
Then there exists $\left(x^{1}, x^{2}, \ldots, x^{n}\right) \in X^{n}$ such that

$$
x^{1}=F\left(x^{1}, x^{2}, \ldots, x^{n}\right), \quad x^{2}=F\left(x^{2}, \ldots, x^{n}, x^{1}\right), \quad \ldots, \quad x^{n}=F\left(x^{n}, x^{1}, \ldots, x^{n-1}\right) .
$$

Moreover, if property (A) is satisfied, then the n-tupled fixed point of $F$ remains unique.

Proof For all $\epsilon>0$, take $\delta(\epsilon)=\left(\frac{1}{k}-1\right) \epsilon$ and apply Corollary 4.1.

Remark 4.2 We replace the continuity hypothesis of $F$ by hypotheses (a) and (b) of Theorem 3.2 , then this result also remains true.

\section{Example}

We give the following example to illustrate our main result.

Example 5.1 Let $X=[0,1]$. Then $(X, \preceq)$ is a partially ordered set under the natural ordering of real numbers. Define $p:[0,1] \times[0,1] \rightarrow \mathbb{R}^{+}$by $p(x, y)=\max \{x, y\}, x, y \in[0,1]$. Then $(X, p)$ is a complete partial metric space.

Now for any fixed even integer $n>1$, consider the product space $X^{n}=[0,1] \times[0,1] \times$ $\cdots \times[0,1], n$ times (in short we write $\left.X^{n}=[0,1]^{n}\right)$. Define $F: X^{n} \rightarrow X$ by

$$
F\left(x^{1}, x^{2}, x^{3}, \ldots, x^{n}\right)=\frac{x^{1}}{n} \quad \text { for } x^{1}, x^{2}, \ldots, x^{n} \in[0,1] .
$$

Then $F$ has the mixed strict monotone property. Also $F$ is a generalized Meir-Keeler type function. The proof follows in two parts, that is, we prove the following:

For $\left(x^{1}, x^{2}, \ldots, x^{n}\right),\left(y^{1}, y^{2}, \ldots, y^{n}\right) \in X^{n}$ with $x^{1} \prec y^{1}, y^{2} \preceq x^{2}, x^{3} \prec y^{3}, \ldots, y^{n} \preceq x^{n}$,

(1) $p\left(F\left(x^{1}, x^{2}, \ldots, x^{n}\right), F\left(y^{1}, y^{2}, \ldots, y^{n}\right)\right)<\max \left\{p\left(x^{1}, y^{1}\right), p\left(x^{2}, y^{2}\right), \ldots, p\left(x^{n}, y^{n}\right)\right\}$,

(2) $\eta\left(\left(F^{m}\left(x^{1}, x^{2}, x^{3}, \ldots, x^{n}\right), F^{m}\left(x^{2}, x^{3}, \ldots, x^{n}, x^{1}\right), \ldots, F^{m}\left(x^{n}, x^{1}, x^{2}, \ldots, x^{n-1}\right)\right)\right.$, $\left.\left(F^{m}\left(y^{1}, y^{2}, y^{3}, \ldots, y^{n}\right), F^{m}\left(y^{2}, y^{3}, \ldots, y^{n}, y^{1}\right), \ldots, F^{m}\left(y^{n}, y^{1}, y^{2}, \ldots, y^{n-1}\right)\right)\right)$

$\rightarrow 0 \quad$ as $m \rightarrow \infty$.

The first part is trivial. For second part, we have

$$
\begin{aligned}
& \eta\left(\left(F^{m}\left(x^{1}, x^{2}, x^{3}, \ldots, x^{n}\right), F^{m}\left(x^{2}, x^{3}, \ldots, x^{n}, x^{1}\right), \ldots, F^{m}\left(x^{n}, x^{1}, x^{2}, \ldots, x^{n-1}\right)\right),\right. \\
&\left.\left(F^{m}\left(y^{1}, y^{2}, y^{3}, \ldots, y^{n}\right), F^{m}\left(y^{2}, y^{3}, \ldots, y^{n}, y^{1}\right), \ldots, F^{m}\left(y^{n}, y^{1}, y^{2}, \ldots, y^{n-1}\right)\right)\right) \\
&= \eta\left(\left(F\left(F^{m-1}\left(x^{1}, x^{2}, \ldots, x^{n}\right), F^{m-1}\left(x^{2}, \ldots, x^{n}, x^{1}\right), \ldots, F^{m-1}\left(x^{n}, x^{1}, \ldots, x^{n-1}\right)\right),\right.\right. \\
& F\left(F^{m-1}\left(x^{2}, \ldots, x^{n}, x^{1}\right), \ldots, F^{m-1}\left(x^{n}, x^{1}, \ldots, x^{n-1}\right), F^{m-1}\left(x^{1}, x^{2}, \ldots, x^{n}\right)\right), \ldots, \\
&\left.F\left(F^{m-1}\left(x^{n}, x^{1}, \ldots, x^{n-1}\right), F^{m-1}\left(x^{1}, x^{2}, \ldots, x^{n}\right), \ldots, F^{m-1}\left(x^{n-1}, \ldots, x^{1}, x^{n}\right)\right)\right), \\
&\left(F\left(F^{m-1}\left(y^{1}, y^{2}, \ldots, y^{n}\right), F^{m-1}\left(y^{2}, \ldots, y^{n}, y^{1}\right), \ldots, F^{m-1}\left(y^{n}, y^{1}, \ldots, y^{n-1}\right)\right),\right. \\
& F\left(F^{m-1}\left(y^{2}, \ldots, y^{n}, y^{1}\right), \ldots, F^{m-1}\left(y^{n}, y^{1}, \ldots, y^{n-1}\right), F^{m-1}\left(y^{1}, y^{2}, \ldots, y^{n}\right)\right), \ldots, \\
&\left.\left.F\left(F^{m-1}\left(y^{n}, y^{1}, \ldots, y^{n-1}\right), F^{m-1}\left(y^{1}, y^{2}, \ldots, y^{n}\right), \ldots, F^{m-1}\left(y^{n-1}, \ldots, y^{1}, y^{n}\right)\right)\right)\right)
\end{aligned}
$$




$$
\begin{aligned}
& =\eta\left(\left(F\left(\frac{x^{1}}{n^{m-1}}, \frac{x^{2}}{n^{m-1}}, \frac{x^{3}}{n^{m-1}}, \ldots, \frac{x^{n}}{n^{m-1}}\right), F\left(\frac{x^{2}}{n^{m-1}}, \frac{x^{3}}{n^{m-1}}, \ldots, \frac{x^{n}}{n^{m-1}}, \frac{x^{1}}{n^{m-1}}\right), \ldots,\right.\right. \\
& \left.F\left(\frac{x^{n}}{n^{m-1}}, \frac{x^{1}}{n^{m-1}}, \frac{x^{2}}{n^{m-1}}, \ldots, \frac{x^{n-1}}{n^{m-1}}\right)\right),\left(F\left(\frac{y^{1}}{n^{m-1}}, \frac{y^{2}}{n^{m-1}}, \frac{y^{3}}{n^{m-1}}, \ldots, \frac{y^{n}}{n^{m-1}}\right),\right. \\
& \left.\left.F\left(\frac{y^{2}}{n^{m-1}}, \frac{y^{3}}{n^{m-1}}, \ldots, \frac{y^{n}}{n^{m-1}}, \frac{y^{1}}{n^{m-1}}\right), \ldots, F\left(\frac{y^{n}}{n^{m-1}}, \frac{y^{1}}{n^{m-1}}, \frac{y^{2}}{n^{m-1}}, \ldots, \frac{y^{n-1}}{n^{m-1}}\right)\right)\right) \\
& =\eta\left(\left(\frac{x^{1}}{n^{m}}, \frac{x^{2}}{n^{m}}, \frac{x^{3}}{n^{m}}, \ldots, \frac{x^{n}}{n^{m}}\right),\left(\frac{y^{1}}{n^{m}}, \frac{y^{2}}{n^{m}}, \frac{y^{3}}{n^{m}}, \ldots, \frac{y^{n}}{n^{m}}\right)\right) \\
& =\max \left\{p\left(\frac{x^{1}}{n^{m}}, \frac{y^{1}}{n^{m}}\right), p\left(\frac{x^{2}}{n^{m}}, \frac{y^{2}}{n^{m}}\right), p\left(\frac{x^{3}}{n^{m}}, \frac{y^{3}}{n^{m}}\right), \ldots, p\left(\frac{x^{n}}{n^{m}}, \frac{y^{n}}{n^{m}}\right)\right\} \\
& =\max \left\{\frac{y^{1}}{n^{m}}, \frac{x^{2}}{n^{m}}, \frac{y^{3}}{n^{m}}, \ldots, \frac{x^{n}}{n^{m}}\right\} \rightarrow 0 \quad \text { as } m \rightarrow \infty \text {. }
\end{aligned}
$$

Hence all the hypotheses of Theorem 3.1 are satisfied. Therefore, $F$ has a unique $n$-tupled fixed point. Here $(0,0, \ldots, 0)$ is an $n$-tupled fixed point of $F$.

\section{Competing interests}

The authors declare that they have no competing interests.

\section{Authors' contributions}

All authors contributed equally in writing this article. They read and approved the final manuscript.

\section{Author details}

'Department of Mathematics, Aligarh Muslim University, Aligarh, 202002, India. ${ }^{2}$ Department of Mathematics, Faculty of Arts and Sciences, Kirikkale University, Yahsihan, Kirikkale 71450, Turkey.

\section{Acknowledgement}

The authors are grateful to the referees for their valuable comments and suggestions which improved the contents of the article.

\section{Received: 29 October 2013 Accepted: 4 April 2014 Published: 09 May 2014}

\section{References}

1. Agarwal, RP, El-Gebeily, MA, O'Regan, D: Generalized contractions in partially ordered metric spaces. Appl. Anal. 87, 1-8 (2008)

2. Altun, I, Damjanovic, B, Djoric, D: Fixed point and common fixed point theorems on ordered cone metric spaces. Appl. Math. Lett. 23, 310-316 (2010)

3. Altun, I, Simsek, H: Some fixed point theorems on ordered metric spaces and application. Fixed Point Theory Appl. 2010, Article ID 621469 (2010)

4. Altun, I, Sola, F, Simsek, H: Generalized contractions on partial metric spaces. Topol. Appl. 157(18), $2778-2785$ (2010)

5. Amini-Harandi, A: Coupled and tripled fixed point theory in partially ordered metric spaces with application to initial value problem. Math. Comput. Model. 57, 2343-2348 (2013)

6. Amini-Harandi, A, Emami, H: A fixed point theorem for contraction type maps in partially ordered metric spaces and application to ordinary differential equations. Nonlinear Anal. 72, 2238-2242 (2010)

7. Aydi, H: Some coupled fixed point results on partial metric spaces. Int. J. Math. Math. Sci. 2011, Article ID 647091 (2011)

8. Aydi, H: Fixed point results for weakly contractive mappings in ordered partial metric spaces. J. Adv. Math. Stud. 4(2), 1-12 (2011)

9. Aydi, $\mathrm{H}$ : Common fixed point results for mappings satisfying $(\varphi, \phi)$-weak contractions in ordered partial metric space. Int. J. Math. Stat. 12(2), 53-64 (2012)

10. Berinde, $\mathrm{V}$ : Generalized coupled fixed point theorems for mixed monotone mappings in partially ordered metric spaces. Nonlinear Anal. 74, 7347-7355 (2011)

11. Berinde, $\mathrm{V}$ : Coupled fixed point theorems for contractive mixed monotone mappings in partially ordered metric spaces. Nonlinear Anal. 75, 3218-3228 (2012)

12. Berinde, V, Pǎcurar, M: Coupled fixed point theorems for generalized symmetric Meir-Keeler contractions in ordered metric spaces. Fixed Point Theory Appl. 2012, 115 (2012)

13. Bhaskar, TG, Lakshmikantham, V: Fixed points theorems in partially ordered metric spaces and applications. Nonlinear Anal. TMA 65, 1379-1393 (2006)

14. Harjani, J, Sadarangani, K: Generalized contractions in partially ordered metric spaces and applications to ordinary differential equations. Nonlinear Anal. 72, 1188-1197 (2010) 
15. Hong, S: Fixed points of multivalued operators in ordered metric spaces with applications. Nonlinear Anal. 72 3929-3942 (2010)

16. Kadelburg, Z, Pavlović, M, Radenović, S: Common fixed point theorems for ordered contractions and quasicontractions in ordered cone metric spaces. Comput. Math. Appl. (2010). doi:10.1016/j.camwa.2010.02.039

17. Lakshmikantham, V, Ćirić, LB: Coupled fixed point theorems for nonlinear contractions in partially ordered metric spaces. Nonlinear Anal. 70, 4341-4349 (2009)

18. Nieto, JJ, Rodríguez-López, R: Contractive mapping theorems in partially ordered sets and applications to ordinary differential equation. Order 22, 223-239 (2005)

19. Nieto, JJ, Rodríguez-López, R: Existence and uniqueness of fixed point in partially ordered sets and applications to ordinary differential equations. Acta Math. Sin. Engl. Ser. 23(12), 2205-2212 (2007)

20. Oltra, S, Valero, O: Banach's fixed point theorem for partial metric spaces. Rend. Ist. Mat. Univ. Trieste 36, 17-26 (2004)

21. O'Regan, D, Petrusel, A: Fixed point theorems for generalized contractions in ordered metric spaces. J. Math. Anal. Appl. 341, 1231-1252 (2008)

22. Ran, ACM, Reurings, MCB: A fixed point theorem in partially ordered sets and some applications to matrix equations. Proc. Am. Math. Soc. 132, 1435-1443 (2004)

23. Rezapour, S, Derafshpour, M, Shahzad, N: Best proximity points of cyclic $\varphi$-contractions in ordered metric spaces. Topol. Methods Nonlinear Anal. 37(1), 193-202 (2010)

24. Romaguera, S, Schellekens, M: Partial metric monoids and semivaluation spaces. Topol. Appl. 153(5-6), 948-962 (2005)

25. Romaguera, S, Valero, O: A quantitative computational model for complete partialmetric spaces via formal balls. Math. Struct. Comput. Sci. 19(3), 541-563 (2009)

26. Romaguera, S: A Kirk type characterization of completeness for partial metric spaces. Fixed Point Theory Appl. 2010 Article ID 493298 (2010)

27. Samet, B: Coupled fixed point theorems for a generalized Meir-Keeler contraction in partially ordered metric spaces. Nonlinear Anal. 72, 4508-4517 (2010)

28. Wolk, ES: Continuous convergence in partially ordered sets. Gen. Topol. Appl. 5, 221-234 (1975)

29. Zhang, X: Fixed point theorems of multivalued monotone mappings in ordered metric spaces. Appl. Math. Lett. 23 235-240 (2010)

30. Cirić, LB, Olatinwo, MO, Gopal, D, Akinbo, G: Coupled fixed point theorems for mappings satisfying a contractive condition of rational type on a partially ordered metric space. Adv. Fixed Point Theory 2(1), 1-8 (2012)

31. Murthy, PP, Rashmi: Tripled common fixed point theorems for $w$-compatible mappings in ordered cone metric spaces. Adv. Fixed Point Theory 2(2), 157-175 (2012)

32. Nashine, HK, Samet, B, Vetro, C: Monotone generalized nonlinear contractions type mappings in ordered metric spaces. Math. Comput. Model. 54, 712-720 (2011)

33. Husain, S, Sahper, H: Generalized $n$-tupled fixed point theorems in partially ordered metric spaces involving an ICS map. Adv. Fixed Point Theory 3(3), 476-492 (2013)

34. Samet, B, Vetro, C: Coupled fixed point, $f$-invariant set and fixed point of $N$-order. Ann. Funct. Anal. 1(2), 4656-4662 (2010)

35. Imdad, M, Soliman, AH, Choudhury, BS, Das, P: On $n$-tupled coincidence and common fixed points results in metric spaces. J. Oper. 2013, Article ID 532867 (2013)

36. Matthews, SG: Partial metric topology. In: Proc. 8th Summer Conference on General Topology and Applications. Ann. New York Acad. Sci., vol. 728, pp. 183-197 (1994)

37. Abdeljawad, T, Karapınar, E, Tas, K: Existence and uniqueness of a common fixed point on partial metric spaces. Appl. Math. Lett. 24, 1900-1904 (2011)

38. Golubović, Z, Kadelburg, Z, Radenović, S: Coupled coincidence points of mappings in ordered partial metric spaces. Abstr. Appl. Anal. 2012, Article ID 192581 (2012). doi:10.1155/2012/192581

39. Haghi, RH, Rezapour, S, Shahzad, N: Be careful on partial metric fixed point results. Topol. Appl. 160, 450-454 (2013)

40. Heckmann, R: Approximation of metric spaces by partial metric spaces. Appl. Categ. Struct. 7, 71-83 (1999)

41. Jleli, M, Karapınar, E, Samet, B: Further remarks on fixed point theorems in the context of partial metric spaces. Abstr. Appl. Anal. 2013, Article ID 715456 (2013)

42. Kadelburg, Z, Nashine, HK, Radenović, S: Coupled fixed point results in 0-complete ordered partial metric spaces. J. Adv. Math. Stud. 6(1), 159-172 (2013)

43. Karapınar, E: Generalizations of Caristi Kirk's theorem on partial metric spaces. Fixed Point Theory Appl. 2011, 4 (2011)

44. Karapınar, E, Erhan, IM: Fixed point theorems for operators on partial metric spaces. Appl. Math. Lett. 24, 1894-1899 (2011)

45. Samet, B, Rajović, M, Lazović, R, Stojiljković, R: Common fixed-point results for nonlinear contractions in ordered partial metric spaces. Fixed Point Theory Appl. 2011, 71 (2011)

46. Samet, B, Vetro, C, Vetro, F: From metric spaces to partial metric spaces. Fixed Point Theory Appl. 2013, 5 (2013). doi:10.1186/1687-1812-2013-5

47. Shatanawi, W, Samet, B, Abbas, M: Coupled fixed point theorems for mixed monotone mappings in ordered partial metric spaces. Math. Comput. Model. (2011). doi:10.1016/j.mcm.2011.08.042

48. Valero, O: On Banach fixed point theorems for partial metric spaces. Appl. Gen. Topol. 6(2), $229-240$ (2005)

49. Erduran, A, Imdad, M: Coupled fixed point theorems for generalized Meir-Keeler contractions in ordered partial metric spaces. J. Nonlinear Anal. Appl. 2012, Article ID jnaa-00169 (2012)

50. Ilic, D, Pavlovic, V, Rakocecic, V: Some new extensions of Banach's contraction principle to partial metric space. Appl. Math. Lett. 24(8), 1326-1330 (2011)

51. Shobkolaei, N, Vaezpour, SM, Sedghi, S: A common fixed point theorem on ordered partial metric spaces. J. Basic Appl. Sci. Res. 1(12), 3433-3439 (2011)

52. Meir, A, Keeler, E: A theorem on contraction mappings. J. Math. Anal. Appl. 28, 326-329 (1969)

53. Suzuki, T: Meir-Keeler contraction of integral type are still Meir-Keeler contractions. Int. J. Math. Math. Sci. 2007, Article ID 39281 (2007). doi:10.1155/2007/39281

54. Suzuki, T: A generalized Banach contraction principle which characterizes metric completeness. Proc. Am. Math. Soc. $136,1861-1869$ (2008)

55. Karapınar, E: Quartet fixed point for nonlinear contraction. arXiv:1106.5472 
10.1186/1687-1812-2014-114

Cite this article as: Imdad et al.: Generalized Meir-Keeler type $n$-tupled fixed point theorems in ordered partial metric spaces. Fixed Point Theory and Applications 2014, 2014:114

Submit your manuscript to a SpringerOpen ${ }^{\circ}$ journal and benefit from:

- Convenient online submission

- Rigorous peer review

- Immediate publication on acceptance

Open access: articles freely available online

- High visibility within the field

- Retaining the copyright to your article

Submit your next manuscript at $\gg$ springeropen.com 